This item was submitted to Loughborough's Research Repository by the author.

Items in Figshare are protected by copyright, with all rights reserved, unless otherwise indicated.

\title{
Measurement of vibrational energy flow in a plate with high energy flow boundary crossing using electronic speckle pattern interferometry
}

PLEASE CITE THE PUBLISHED VERSION

http://dx.doi.org/10.1016/j.apacoust.2012.04.002

PUBLISHER

(c) Elsevier

VERSION

AM (Accepted Manuscript)

LICENCE

CC BY-NC-ND 4.0

\section{REPOSITORY RECORD}

Eck, Thomas, and Stephen J. Walsh. 2012. "Measurement of Vibrational Energy Flow in a Plate with High Energy Flow Boundary Crossing Using Electronic Speckle Pattern Interferometry”. figshare.

https://hdl.handle.net/2134/9905. 
This item was submitted to Loughborough's Institutional Repository (https://dspace.lboro.ac.uk/) by the author and is made available under the following Creative Commons Licence conditions.

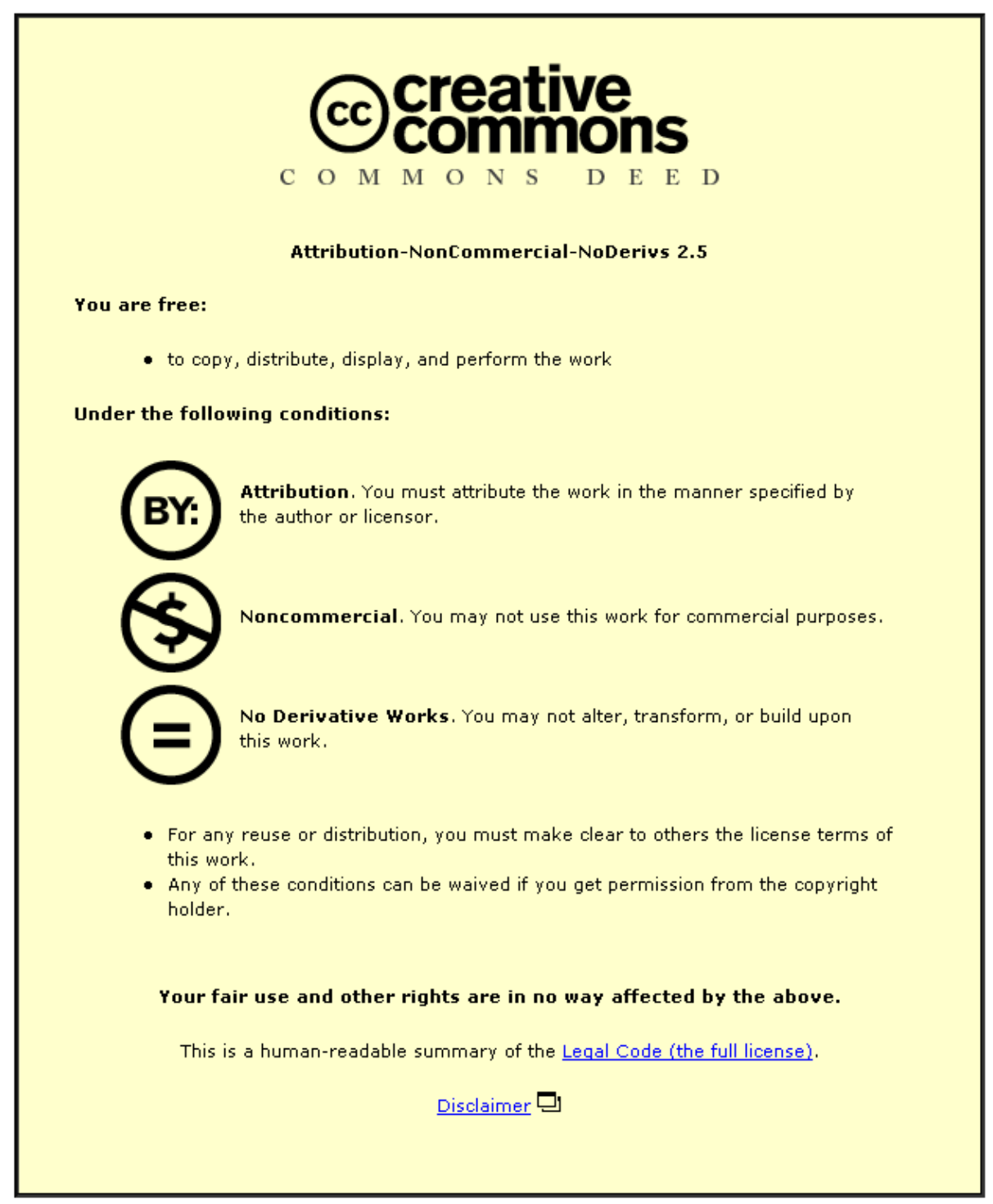

For the full text of this licence, please go to: http://creativecommons.org/licenses/by-nc-nd/2.5/ 


\title{
Measurement of vibrational energy flow in a plate with high energy flow boundary crossing using electronic speckle pattern interferometry
}

\author{
T. Eck and S.J. Walsh \\ Department of Aeronautical and Automotive Engineering, \\ Loughborough University, Loughborough, LE11 3TU, UK \\ Corresponding author. Tel.: +44 (1509) 227208 \\ E-mail address: S.J.Walsh@Lboro.ac.uk (S.J. Walsh)
}

\begin{abstract}
The measurement of vibrational energy flow is an important tool in understanding the vibrational behaviour of structures. In the past, because of transducer constraints, the measurement of vibrational energy flow was mostly restricted to single point measurements. However, recent developments in advanced laser measurement techniques, such as electronic speckle pattern interferometry (ESPI), have gained interest in applying two-dimensional, multi-point measurement techniques to the estimation of vibrational energy flow. This paper addresses the measurement of vibrational energy flow in a plate by using an ESPI based vibrational energy flow measurement technique. A radially symmetric bending wave plate vibration model is introduced and theoretical expressions for energybased quantities are derived. To assess the accuracy of the measurement method, these theoretical quantities are compared to synthetic results derived from the ESPI energy flow measurement technique. The ESPI measurement technique is also applied to an experimental 'infinite' plate. Thus, a specially designed experimental apparatus was constructed so as to minimise undesired wave reflections in the plate and, thus, achieve a high energy flow boundary crossing at the edges of the plate. To reduce the effect of optical noise contamination on the ESPI measured out-of-plane plate displacement data, optimal filters were applied prior to the vibrational energy flow computation. To appraise the accuracy of the experimental method, measured vibrational power on the plate is compared with measured vibrational input power. A difference of less than $1 \mathrm{~dB}$ between both quantities indicates that vibrational energy flow within a rectangular plate that contains radially symmetric wave propagation can be measured to a good degree of accuracy if appropriate filtering is applied.
\end{abstract}

Keywords: Structural vibration; infinite plate; vibrational energy flow; wavenumber domain filtering; electronic speckle pattern interferometry. 


\section{Introduction}

Early work on the measurement of vibrational energy flow using biaxial accelerometers was reported by Noiseux in 1970 [1]. However, Noiseux's attempt to measure vibrational power was limited by the lack of measurement equipment at that time. In 1976 Pavić [2] introduced a wave intensity measurement method using an array of velocity transducers measuring in the far and nearfield. Using a finite difference approximation to obtain the spatial derivatives, the power flow distribution in the structure when in flexure was determined. Verheij [3] proposed an accelerometer-based cross-spectral density method to measure one-dimensional power flow for bending, longitudinal, and torsional waves. The cross-spectral density method is now widely employed as a standard power flow measurement technique. However, accelerometer-based power flow measurements and the usage of a finite difference approximation suffer from a number of drawbacks. Most mentionable are the influence on the structure's dynamic response due to added transducer masses, unwanted phase mismatch between two or more transducer channels and the errors of the finite difference approximation [4]. Thus, alternative measurement and processing techniques are desired in order to overcome the limitations of the conventional accelerometer-based method.

An alternative approach to the finite difference approximation is the wavenumber domain approach that was suggested by Williams, Dardy, and Fink in Ref. [5]. This approach was an outgrowth from nearfield acoustic holography [6]. In Ref. [5] the acoustic pressure in a plane close to the surface of a vibrating plate was measured. To obtain the spatial derivatives of the plate's surface velocity a technique relying on the spatial Fourier transform was applied and was denoted as a wavenumber domain approach. The concept of the wavenumber domain approach to compute spatial derivatives of a structure's surface displacement or velocity was applied analytically to a beam in flexure by Unglenieks and Bernard [7]. In Ref. [7], the wavenumber domain approach was addressed as a spatial derivative technique. Morikawa et al. [8] denoted this technique as a $k$-space method. Morikawa et al. [8] showed that the primary advantage of employing the spatial derivative technique, in contrast to the finite difference approximation technique, is the ability to handle noise corrupted data. Since noise in the acquired signal will be amplified during the derivation process in the $k$-space or wavenumber domain, the authors removed these noise components by using a low-pass filter. Problems using the spatial derivative technique arose due to spectral leakage when a non-integer number of wavelengths from the signal were analysed. Nejade and Singh [9] applied a two-dimensional spatial Fourier transform in combination with a $k$-space filtering procedure to compute flexural intensity in plates from a grid of lightweight accelerometers. The authors employed an ideal band-pass filter, centred at the wavenumber of the vibrating structure, to remove noisy wavenumber components.

Pascal et. al. [10-12] applied holographic interferometry to measure structural intensity in a plate by measuring the out-of-plane velocity of the whole vibration field. For comparison reasons an energy transfer indicator was employed to compare measured intensity with the theoretical intensity present in an infinite plate due to flexural wave propagation. A 2D-FIR filter was used to reduce measurement noise. Additionally, a lowpass filter was applied in the wavenumber domain to minimise the noise amplification during the derivation process. Alves et al. [13] measured bending wave intensity in a free- 
free plate using a double pulsed ruby-laser to illuminate the vibrating object. Spatial derivatives were computed by employing regressive discrete Fourier series [14] in the wavenumber domain. A smoothing technique using a two-dimensional discrete Fourier series was applied and noise spikes were removed by using a median filter. Results were compared with computed intensity calculated using finite element modelling. Despite good agreement in some cases no comparison to conventionally measured power data were given.

Chambard et al. [15] applied TV-Holography to a number of different mechanical structures. With the use of specially developed software called VIPART [16] amplitude, phase, intensity, divergence and force distribution maps were produced. Image processing of the holographic data enabled the correction of amplitude and phase data if geometrical distortions were present. Eck et al. [17] applied Electronic speckle Pattern Interferometry (ESPI) to measure vibrational power in an 'infinite' experimental beam. The wavenumber processing technique was employed to determine the spatial derivatives required for energy flow computation. Comparisons with conventionally acquired input power and transmitted power made using single-point transducers were made. It was shown that ESPI measurements made at non-resonant frequencies showed good agreement with the conventionally measured vibrational power data. However, at local maxima of the frequency response function energy flow measurement errors occurred.

This paper presents a theoretical, numerical and experimental investigation of the vibrational energy flow in a point force excited 'infinite' plate made using ESPI measurement system. For ease of reference, this processing technique will be referred to as VEFESPI. In Section 2 equations for transmitted vibrational power in an infinite plate in both polar and Cartesian coordinates are derived. In an extension of existing work simplified expressions for the transmitted vibrational power are developed using an approximation of the Hankel function representation of the displacement field. In Section 3 a numerical analysis of the VEFESPI method is conducted by using synthetically generated plate displacement data. These results are compared to the theoretical expressions developed in Section 2 and to a calculation of the total power input to the structure. In Section 4 the experimental apparatus and measurement method are described. Conventional single point transducer measurements were undertaken at the excitation location in order to obtain point mobility and input power data. Also in Section 4 optimum filter parameters are derived from an analysis of synthetically generated infinite plate displacements and superimposed ESPI noise. These optimum filter parameters are used to minimise the effect of noise when determining vibrational energy flow from the measured out-of-plane ESPI displacements. Section 5 presents results obtained from the measurement of vibrational energy flow in an experimental 'infinite' plate. To assess the accuracy of the ESPI based vibrational energy flow measurement (VEFESPI) method, an energy balance analysis is carried out by comparing the real part of the total transmitted vibrational power on the plate to the measured input power value.

\section{Theory of vibrational energy flow in an 'infinite' plate}

\subsection{Vibrational energy flow in a plate}


Vibrational energy flow within a thin plate is caused by the internal action of three individual energy flow mechanisms. A plate under lateral load excitation responds internally by shear forces, internal bending moments and internal twisting moments. Timeaveraged vibrational power per unit length in the $x$ and $y$ directions of a plate may be written as [2]:

$$
\begin{aligned}
& \overline{P_{x}}=\frac{D}{2}\left(\begin{array}{l}
\frac{\partial}{\partial x}\left(\nabla^{2} w\right)\left(\frac{\partial w}{\partial t}\right)^{*}-\left(\frac{\partial^{2} w}{\partial x^{2}}+\nu \frac{\partial^{2} w}{\partial y^{2}}\right)\left(\frac{\partial^{2} w}{\partial x \partial t}\right)^{*} \\
-(1-\nu) \frac{\partial^{2} w}{\partial x \partial y}\left(\frac{\partial^{2} w}{\partial y \partial t}\right)^{*}
\end{array}\right), \\
& \bar{P}_{y}=\frac{D}{2}\left(\begin{array}{l}
\left.\frac{\partial}{\partial y}\left(\nabla^{2} w\right)\left(\frac{\partial w}{\partial \mathrm{t}}\right)^{*}-\left(\frac{\partial^{2} w}{\partial y^{2}}+\nu \frac{\partial^{2} w}{\partial x^{2}}\right)\left(\frac{\partial^{2} w}{\partial y \partial \mathrm{t}}\right)^{*}\right) . \\
-(1-\nu) \frac{\partial^{2} w}{\partial x \partial y}\left(\frac{\partial^{2} w}{\partial x \partial \mathrm{t}}\right)^{*}
\end{array}\right) .
\end{aligned}
$$

Herein, $w$ denotes the complex plate displacement and the over bar denotes a quantity per unit length. Further, $\nabla^{2}=\partial^{2} / \partial x^{2}+\partial^{2} / \partial y^{2}$ is the two-dimensional Laplace operator, $D=$ $E h^{3} / 12\left(1-v^{2}\right)$ is denoted as the plate's flexural rigidity, whereas $E$ is Young's modulus, $h$ is the plate's thickness and $v$ is Poisson's ratio. It can be seen that the product of shear force, bending moment and twisting moment in combination with their respective velocities form a vibrational power per unit length expression. Here, the real part of Eqs. (1) and (2) is denoted as active vibrational energy flow per unit length (in $\mathrm{Wm}^{-1}$ ). The imaginary part of Eqs. (1) and (2) is often denoted as reactive vibrational energy flow per unit length (in $\mathrm{Wm}^{-1}$ ). However, since the physical meaning of the reactive energy flow is not clear, it will not be considered further in this analysis.

Transverse point force excitation of an infinite plate results in circular wave motion. Thus, it is more convenient to describe the energy flow in polar coordinates. The Cartesian coordinates $x$ and $y$ are then a function of the radius $r$ and the angle $\theta$. With the polar Laplace operator $\nabla^{2}=\partial^{2} / \partial r^{2}+r^{-1} \partial / \partial r+r^{-2} \partial^{2} / \partial \theta^{2}$, Eqs. (1) and (2) can be written in polar form as:

$$
\overline{P_{r}}=\frac{D}{2}\left[\begin{array}{l}
\frac{\partial}{\partial r}\left(\nabla^{2} w\right)\left(\frac{\partial w}{\partial \mathrm{t}}\right)^{*}-\left(\frac{\partial^{2} w}{\partial r^{2}}+\nu\left(\frac{1}{r} \frac{\partial w}{\partial r}+\frac{1}{r^{2}} \frac{\partial^{2} w}{\partial \theta^{2}}\right)\right)\left(\frac{\partial^{2} w}{\partial r \partial t}\right)^{*}- \\
(1-\nu) \frac{\partial}{\partial r}\left(\frac{1}{r} \frac{\partial w}{\partial \theta}\right)\left(\frac{\partial^{2} w}{\partial \theta \partial t}\right)^{*}
\end{array}\right]
$$




$$
\overline{P_{\theta}}=\frac{D}{2}\left[\begin{array}{l}
\frac{1}{r} \frac{\partial}{\partial \theta}\left(\nabla^{2} w\right)\left(\frac{\partial w}{\partial \mathrm{t}}\right)^{*}-\left(\frac{1}{r} \frac{\partial w}{\partial r}+\frac{1}{r^{2}} \frac{\partial^{2} w}{\partial \theta^{2}}+\nu \frac{\partial^{2} w}{\partial r^{2}}\right)\left(\frac{\partial^{2} w}{\partial \theta \partial t}\right)^{*}- \\
(1-\nu) \frac{\partial}{\partial r}\left(\frac{1}{r} \frac{\partial w}{\partial \theta}\right)\left(\frac{\partial^{2} w}{\partial r \partial t}\right)^{*}
\end{array}\right]
$$

Due to the complex displacement, $w$, a complex energy transmission per unit length is assumed and, hence, the plate's time-averaged vibrational power per unit length vector field is given as:

$$
\vec{P}=\left\{\begin{array}{c}
\Re\left\{\overline{P_{x}}\right\}+\mathrm{j} \Im\left\{\overline{P_{x}}\right\} \\
\Re\left\{\overline{P_{y}}\right\}+\mathrm{j} \Im\left\{\overline{P_{y}}\right\}
\end{array}\right\} .
$$

Note, although $\overline{P_{x}}$ and $\overline{P_{y}}$ are both two-dimensional complex vector fields the subscript is used only to indicate the direction of the energy that flows into the $x$ and $y$ direction, respectively.

\subsection{Energy balance and vibrational input power}

The energy balance of an infinite plate structure can be approximated very well by neglecting the dissipated energy that is lost due to internal friction or sound radiation. When neglecting the dissipation process, the real part of the total transmitted vibrational power is in equilibrium with the real part of the complex vibrational input power, $\left\langle P_{i n}\right\rangle_{t}$, injected into the plate. This can be expressed as [18]:

$$
\left\langle P_{i n}\right\rangle_{t}=-\oint_{l}\langle\overrightarrow{\vec{P}}\rangle_{t} d l .
$$

Here, $l$ is a closed contour on the plate's surface. The negative sign in Eq. (6) indicates an energy outflow, i.e. vibrational energy flows away from the excitation location.

Vibrational input power of the infinite plate is derived by using the well-known point mobility of an infinite plate that is defined as [21]:

$$
Y_{F_{\infty}}=\frac{\omega}{8 D k^{2}}=\frac{1}{8 \sqrt{D \rho h}} .
$$

Where the subscript $F_{\infty}$ indicates force excitation of an infinite plate. The vibrational input power to an infinite plate when excited by a transverse harmonic point force of amplitude $F_{0}$ and response velocity $v_{0}$ at the excitation location can be written as: 


$$
\begin{aligned}
& \left(P_{\text {in }}\right)_{F_{\infty}}=\frac{F_{0}^{2} \omega}{16 D k^{2}}=\frac{F_{0}^{2}}{16 \sqrt{D \rho h}}, \\
& \left(P_{\text {in }}\right)_{F_{\infty}}=4\left|v_{0}\right|^{2} \sqrt{D \rho h}
\end{aligned}
$$

\subsection{Transmitted vibrational energy using asymptotic expansion functions}

Inspection of Eqs. (3) and (4) shows that vibrational power within a plate can be formed by substituting partial derivatives of the plate's surface displacement into the respective vibrational energy flow equations. The complex, polar and transverse response of the infinite plate to a harmonic point force excitation is given by [21]:

$$
w(r, \theta, t)=\frac{F_{0}}{\mathrm{j} 8 D k^{2}}\left(H_{0}^{(2)}(k r)-H_{0}^{(2)}(-\mathrm{j} k r)\right) e^{\mathrm{j} \omega t} .
$$

Here, $F_{0}$ is the magnitude of the excitation force, $k$ is the plate's flexural wavenumber and $r$ is the radial distance from the excitation location. It can be seen from Eq. (10) that the displacement is formed by the difference of two Hankel functions of the second kind and of zero order. The Hankel function $H_{0}^{(2)}(k r)$ represents a travelling farfield wave component and the Hankel function $H_{0}^{(2)}(-\mathrm{j} k r)$ represents a decaying nearfield wave component around the excitation location. Lande and Langley [20] have derived expressions for total transmitted vibrational power due to cylindrical bending waves in plates using either modified Bessel functions or Hankel functions to model the displacement. In cases where the inward propagating and the outward propagating wave components are described by second order Hankel functions it was shown in Ref. [20] that there is no interactive energy flow between the wave components. It was also noted that in Ref. [20] that there is no interactive energy flow between propagating and evanescent waves.

\subsubsection{Polar expressions}

This study is primarily interested in the energy flow in the vibrational farfield of the plate. Thus, to simplify the vibrational energy derivations, the Hankel function difference, as shown in Eq. (10), can be approximated by truncating the asymptotic expansion of the

Hankel functions. For large arguments, $|k r| \gg 1$, the polar approximation of the infinite plate displacement is given by [21]:

$$
w(r, \theta, t) \approx \frac{F_{0}}{\mathrm{j} 8 D k^{2}} \sqrt{\frac{2}{\pi k r}}\left(e^{-\mathrm{j}\left(k r-\frac{\pi}{4}\right)}-\mathrm{j} e^{-k r}\right) e^{\mathrm{j} \omega t} .
$$

In Ref. [21] it was shown that for $|k r| \geq 4$ very small errors can be expected. The first exponential term in Eq. (11) represents a travelling wave component and the second exponential term represents an evanescent wave component. 
If one substitutes Eq. (11) into Eqs. (3) and (4), polar vibrational power expressions of the infinite plate may be obtained. It can be realised that the infinite plate displacement has only radial and temporal dependency. The displacement does not alter angularly. Thus, Eq. (4) and the angular derivatives in Eq. (3) can be neglected in the vibrational energy flow analysis. A complete derivation of vibrational power from the truncated asymptotic expansion for large values of $|k r|$ of an infinite plate is given in Ref. [18]. From Ref. [18] the active vibrational energy flow per unit length of the infinite plate can be found to be:

$$
\left(\overline{P_{r_{a}}}\right)_{F_{\infty}}=\frac{F_{0}^{2} \omega}{512 D \pi}\left(\frac{16}{k^{2} r}-\frac{4}{k^{4} r^{3}}\right) .
$$

The subscript $a$ denotes an active energy quantity. Eq. (12) displays the active vibrational energy flow of an infinite plate in dependency upon the radius $r$ when modelling the point forced infinite plate response by Eq. (11). It was noted in Ref. [18] that only the travelling wave component is contributing to active vibrational energy flow. The net energy flow per unit length can also be obtained from the equation for the power input to the plate, Eq. (8), by division by by $2 \pi r$. Inspection of Eq. (12) shows that the first term of Eq. (12) is equivalent to the net energy flow per unit length and, thus, the second term of Eq. (12) can be identified as the error made when using the polar approximation of the infinite plate displacement, Eq. (11).

A simple energy balance can be computed for the infinite plate by using Eq. (6). Thus, the integrated active vibrational energy flow per unit length of the infinite plate around a closed contour is equal to the real part of the complex, time-averaged vibrational input power injected into the structure. Hence:

$$
\Re\left\{\left\langle\underline{P}_{i n}\right\rangle_{t}\right\}=\oint_{C}\left(\overline{P_{r_{a}}}\right)_{F_{\infty}} d r .
$$

When dealing with polar expressions, ideally a circle may be chosen as the shape of the contour to be integrated. Fig. 1 displays the circular and the rectangular contour shapes employed in this work. If one substitutes the active vibrational energy flow expression given by Eq. (12) into Eq. (13) and takes into account that $d r=r d \phi$, one may obtain the following integral expression:

$$
\left(P_{a}\right)_{F_{\infty}}=\frac{F_{0}^{2} \omega}{512 D \pi} \int_{0}^{2 \pi}\left(\frac{16}{k^{2}}-\frac{4}{k^{4} r^{2}}\right) d \phi .
$$

By solving Eq. (14) the total transmitted vibrational power is:

$$
\left(P_{a}\right)_{F_{\infty}}=\frac{F_{0}^{2} \omega}{16 D k^{2}}\left(1-\frac{1}{4 k^{2} r^{2}}\right) .
$$


From Eq. (15) it can be noticed that the first term is equal to the vibrational input power given by Eq. (8). The second term can be identified as the error made when using the truncated exponential asymptotic expansion of the Hankel functions. However, it can also be seen from Eq. (15) that this error decreases quadratically with increasing radius $r$. Thus, for large arguments of $|k r|$, the error introduced by the approximation can be assumed to be small.

\subsubsection{Cartesian expressions}

Using the relation $r^{2}=x^{2}+y^{2}$ the polar approximation of the infinite plate displacement, as shown by Eq. (11), can be written in Cartesian coordinates as:

$$
w(x, y, t) \approx \frac{F_{0}}{\mathrm{j} 8 D k^{2}} \sqrt{\frac{2}{\pi k\left(x^{2}+y^{2}\right)^{\frac{1}{2}}}}\left(e^{-\mathrm{j}\left(k\left(x^{2}+y^{2}\right)^{\frac{1}{2}}-\frac{\pi}{4}\right)}-\mathrm{j} e^{-k\left(x^{2}+y^{2}\right)^{\frac{1}{2}}}\right) e^{\mathrm{j} \omega t} .
$$

Time-averaged vibrational power in the $x$ direction that is defined by Cartesian coordinates can be found by substituting Eq. (16) into Eq. (1). The active vibrational energy flow in the $x$ direction only is given as:

$$
\left(\overline{P_{x_{a}}}\right)_{F_{\infty}}=\frac{F_{0}^{2} \omega x}{512 D \pi}\left(\frac{16}{k^{2}\left(x^{2}+y^{2}\right)}-\frac{4}{k^{4}\left(x^{2}+y^{2}\right)^{2}}\right) \text {. }
$$

The active vibrational energy flow of the infinite plate in the $y$ direction is obtainable by interchanging the spatial variables $x$ and $y$. It can be seen from Eq. (17) that only the variable $x$ in front of the bracket term needs to be substituted by the variable $y$ in order to find the active vibrational energy flow in the $y$ direction. Note that the vectors $\left(\overline{P_{x_{a}}}\right)_{F_{\infty}}$ and $\left(\overline{P_{y_{a}}}\right)_{F_{\infty}}$ can also be formed from Eq. (12) by resolving $\left(\overline{P_{r_{a}}}\right)_{F_{\infty}}$ into the $x$ and $y$ directions. As with Eq. (12), inspection of Eq. (17) shows that the first term of Eq. (17) is equivalent to the net energy flow per unit length and, thus, the second term of Eq. (17) can be identified as the error made when using the polar approximation of the infinite plate displacement, Eq. (16).

The total transmitted vibrational power can be found by integrating Eq. (17) and its equivalent expression for the $y$ direction along a closed square contour, as shown by Fig. 1. Hence, the total transmitted vibrational energy within the infinite plate can be determined as:

$$
\left(P_{a}\right)_{F_{\infty}}=\frac{F_{0}^{2} \omega}{16 D k^{2}}\left(1-\frac{1}{4 k^{2} r_{c}^{2}}\left(\frac{1}{\pi}+\frac{1}{2}\right)\right) .
$$


It can be realised from Eq. (18) that the first term is equal to the vibrational input power, as given by Eq. (8). The second term in Eq. (18) is the error made due to the asymptotic displacement approximation and the closed square contour integration. This term, in comparison to the error term shown by Eq. (15), has been reduced by the product $\left(\frac{1}{\pi}+\frac{1}{2}\right)$. A complete derivation of vibrational energy flow within the infinite plate, when using Cartesian coordinates, is given in Ref. [18].

\section{Numerical vibrational energy flow computation}

This section investigates vibrational energy flow determination in an infinite plate structure by inserting artificially generated infinite plate displacement data into the VEFESPI computation method. The results of the VEFESPI analysis are then compared to the theoretical formulae for the active vibrational energy flow per unit length and the total transmitted power derived in Section 2.

\subsection{The VEFESPI method}

The VEFESPI method calculates the vector field of complex time-averaged vibrational power per unit length in a plate, Eq. (5), by applying Eqs. (1) and (2) to a Cartesian coordinate set of displacement data. As shown by Eqs. (1) and (2) vibrational power based quantities are described by partial spatial derivatives of the plate's transverse displacement. The VEFESPI method uses wavenumber domain processing in order to obtain the required spatial derivatives. This technique is addressed here as a $k$-space derivation (KSD) method. Fig. 2 displays the entire VEFESPI method consisting of the data acquisition block, the $k$-space derivation method and the computation of vibrational power based quantities. A detailed description of the method as applied to one-dimensional structures is given in Ref. [17].

The KSD method can be divided into four steps, as shown in Fig. 2. In the first step the spatially tabulated displacement data $u(x, y ; \omega)$ are transformed into the wavenumber domain by applying the forward two-dimensional spatial Fourier transform:

$$
U\left(k_{x}, k_{y} ; \omega\right)=F\{u(x, y ; \omega)\}=\frac{1}{4 \pi^{2}} \int_{-\infty}^{\infty} \int_{-\infty}^{\infty} u(x, y ; \omega) e^{-\mathrm{j}\left(k_{x} x+k_{y} y\right)} d x d y
$$

Herein, $F$ denotes a forward Fourier transform. Within the wavenumber domain $k$-space filtering is employed. Thus, a multiplication of the wavenumber spectrum $U\left(k_{x}, k_{y} ; \omega\right)$ with the respective wavenumber vectors $K_{x}$ and $K_{y}$ is carried out. Fourier transforming these spectral derivatives back into the spatial domain will complete the process. The inverse two-dimensional spatial Fourier transform can be expressed as:

$$
u(x, y ; \omega)=F^{-1}\left\{U\left(k_{x}, k_{y} ; \omega\right)\right\}=\int_{-\infty}^{\infty} \int_{-\infty}^{\infty} U\left(k_{x}, k_{y} ; \omega\right) e^{j\left(k_{x} x+k_{y} y\right)} d k_{x} d k_{y}
$$


where $F^{-1}$ denotes an inverse Fourier transform. The entire $k$-space derivation method can be expressed as:

$$
\frac{\partial^{m} \partial^{n}}{\partial x^{m} \partial y^{n}}[u(x, y)]=F^{-1}\left\{\left(\mathrm{j} K_{x}\right)^{m}\left(\mathrm{j} K_{y}\right)^{n} F\{u(x, y)\}\right\} .
$$

The order of the respective spatial derivative is denoted by integers $m$ and $n$, respectively. The $k$-space derivation method is potentially more accurate than the finite difference approximation [1-3]. However a drawback of this method stems from the windowing effect due to Fourier transforming periodic signals consisting of a non-integer number of periods [12]. In this case spectral leakage will affect the accuracy of the resulting spectrum. This leakage error will be amplified when multiplying in the wavenumber domain to compute the required spectral derivatives. Measures to reduce this effect, for example by using wavenumber domain filtering, are discussed in the next section.

\subsection{Prediction of vibrational energy flow in an infinite plate using a Hankel function approximation}

The point force excited infinite plate response is a radially symmetric wave that is travelling away from the excitation location. For farfield applications, the exponential asymptotic expansion of the Hankel functions is implemented. Thus, the forced infinite plate response is generated by numerically evaluating Eq. (11) using a square matrix of $128^{*} 128$ data points in the $x$ and $y$ directions. A unity excitation force of $F_{0}=1 \mathrm{~N}$ is applied. The infinite plate displacement is investigated within a spatial window of dimensions $L_{x}=L_{y}=1 \mathrm{~m}$ using an excitation frequency of $f_{0}=986.7 \mathrm{~Hz}(k=20 \pi \mathrm{rad}$ $\mathrm{m}^{-1}$ ). The material properties of the simulated infinite plate are shown in Table 1 . The error from the exponential approximation of the Hankel function is negligible from $|k r| \geq 4$ onwards [21]. Therefore, with locations greater than $r=1 / 5 \pi \mathrm{m}(6.4 \mathrm{~cm})$ from the excitation location Eq. (11) is a good approximation of the displacement. Due to the radial nature of the displacement signal combined with a square Cartesian coordinate data matrix, an integer number of wavelenghts cannot be included in the displacement signal window to be Fourier transformed. Thus, the use of a non-integer number of wavelengths in the plate displacement signal cannot be avoided. Furthermore, as evident from Eq. (11), a nearfield component is also present. For these two reasons vibrational energy flow computation errors due to the undesired alteration of the spectral components in the wavenumber domain when computing spatial derivatives can be expected.

Fig. 3a displays the modulus of the $3^{\text {rd }}$ partial derivative of the wavenumber matrix $\partial^{3} W\left(k_{x}, k_{y}\right) / \partial k_{x}^{3}$ determined by the VEFESPI method. For comparison, the exact value of the $3^{\text {rd }}$ spatial derivative of the displacement calculated by analytically differentiating and then numerically Fourier transforming Eq. (11) is shown in Fig 3b. The one-dimensional derivative cross-sections at $k_{y}=0$ are shown in Figs. 3c-d. It can be seen from Fig. 3 that undesired oscillations are introduced into the VEFESPI $3^{\text {rd }}$ partial derivative signal $\partial^{3} W\left(k_{x}, k_{y}\right) / \partial k_{x}^{3}$ due to signal's non-integer number of wavelengths. These undesired 
oscillations arise due to spectral leakage amplification in the wavenumber multiplication process when computing the spatial derivatives using Eq. (21). However, these undesired oscillations can be reduced by approximating the oscillating vibrational energy flow curve using a 'curve wrap' method. The curve wrap method is illustrated in Appendix A and described in more detail in Ref. [18].

Fig. 4 displays the active vibrational energy flow map of the infinite plate displacement computed by the VEFESPI + curve wrap method. For better illustration the numbers of data points per length were reduced from 128 to 16, simply by averaging the original vibrational energy flow maps over distance. Furthermore, the active vibrational energy flow vectors within the location of $|k r| \leq 4$ were set to zero because for these regions the asymptotic expansion equations, Eqs. (11) and (16), are not valid. The vectors show energy flow per unit length/width and are, thus, in units of $\mathrm{W} / \mathrm{m}$. It can be seen from Fig. 4 that the active vibrational energy flows radially and symmetrically away from the excitation location. It can also be noticed that with increasing distance $r$ the magnitude of active vibrational energy flow is decreasing in order to obey the energy conservation law.

To determine the accuracy of the VEFESPI + curve wrap method when computing vibrational energy flow within infinite plates, a two-dimensional relative error distribution $\Delta \varepsilon_{x}$ was calculated from the determined VEFESPI + curve wrap active vibrational energy flow and the theoretical vibrational energy flow solution in the $x$ direction as: given by Eq. (17). Thus,

$$
\Delta \varepsilon_{x}=\left(1-\frac{\left(\overline{P_{x}}\right)_{V E F E S P I}}{\left(\overline{P_{x}}\right)_{\text {exact }}}\right) \cdot 100 \% .
$$

Note, the relative error in the $y$ direction $\Delta \varepsilon_{y}$ is calculated simply by substituting subscript $x$ with subscript $y$ in Eq. (22). Fig. 5 displays the relative active vibrational energy flow error distribution. For better illustration, the number of data points per length was reduced from 128 to 32 . Furthermore, the active vibrational energy flow values within the region of $|k r| \leq 4$ were also set to zero. It can be seen from Fig. 5 that the relative error made when computing the active vibrational energy flow by the VEFESPI + curve wrap method is less than $0.5 \%$.

An alternative method to assess the accuracy of the VEFESPI + curve wrap method is via the numerical contour integration of the two-dimensional energy fields $\overline{P_{x}}$ and $\overline{P_{y}}$ using an energy balance relationship. It was shown by Eq. (14) that integration of the active vibrational energy flow around a closed contour is equal to the real part of the vibrational input power injected into the plate structure. Fig. 6 displays the comparison of total transmitted vibrational power versus the vibrational input power. A Cartesian coordinate based numerical contour integration was carried out for each location $|k r|$ by numerically evaluating the active vibrational energy flow per unit length in the $x$ and $y$ directions around a square contour, as shown in Fig. 1. With a sample number of 128 for each side length, 64 concentric squares were integrated numerically. It can be seen from Fig. 6 that at Helmholtz numbers greater than $|k r| \approx 5$ the total transmitted vibrational 
power $\left(P_{a}\right)_{F_{\infty}}$ within the infinite plate is approximately equal to the input power $\left(P_{i n}\right)_{F_{\infty}}$ calculated using Eq. (8). At values of $|k r|<5$ the trend follows that expected by the error term in Eq. (18). For example, at a value of $|k r| \approx 5$, a $1 \%$ difference between the vibrational input power and the total transmitted vibrational power is predicted by Eq. (18). Whereas at a value of $|k r| \approx 1$, a $25 \%$ difference is predicted by Eq. (18). Thus, the discrepancy appears to have arisen because of the approximate expression for the simulated plate displacement and not because of the signal processing involved.

\section{Experimental apparatus and measurement method}

\subsection{Experimental apparatus}

To measure vibrational energy flow, a point force excited 'infinite' plate was designed, as shown in Fig. 7. The experimental apparatus consisted of an upper and lower frame, which enclosed the rectangular plate specimen. The rectangular plate was made from mild steel, $1300 \mathrm{~mm}$ in length, $1100 \mathrm{~mm}$ in width and $1 \mathrm{~mm}$ in thickness. Between the inner and outer frames, kiln dried sand and foam wedges were inserted in order to provide an anechoic termination. The plate was embedded between the inner and outer frame stretching $192 \mathrm{~mm}$ into the anechoic termination region. The length of $192 \mathrm{~mm}$ ensured that approximately one spatial wavelength of the lowest frequency to be measured was covered in sand. The lowest frequency capable of being measured was about $400 \mathrm{~Hz}$, due to limitations of the ESPI measurement system. The inner dimensions of the frame were chosen to be in a ratio of $4: 3$, exactly the same as the aspect ratio of the CCD camera. A region of $700 \mathrm{~mm}$ by $525 \mathrm{~mm}$ of the plate was illuminated by the continuous $150 \mathrm{~mW}$ frequency doubled Nd:Yag laser. This region will be referred to as the ESPI window. The plate was excited by an electro-dynamic exciter, off-centre at a position $x_{0}=$ $550 \mathrm{~mm}$ and $y_{0}=600 \mathrm{~mm}$, measured from the left-hand corner of the plate.

\subsection{Measurement of point mobility, vibrational input power and ESPI plate displacement}

The point mobility of the 'infinite' plate was measured prior to the ESPI measurement using an impact hammer, response accelerometer and the standard $\mathrm{H} 1$ estimator. During the ESPI measurement power input to the plate was also measured. Usually, vibrational input power is measured using the imaginary part of the cross-spectral density between the driving force and the resulting acceleration. However, during the experiment an erroneous cross-spectral density signal between the driving force and the resulting acceleration was recorded. Thus, input power was calculated by using two alternative methods: (i) by appling Eq. (9), the product of the theoretical impedance of an infinite plate and the measured response velocity squared at the excitation location; and (ii) from the product of the real part of the measured point impedance of the plate and the measured response velocity squared.

The transverse time-averaged ESPI measured complex plate displacement is generated as: 


$$
w(x, y)_{E S P I}=A(x, y) e^{j \phi(x, y)} .
$$

Herein, $A(x, y)$ is the measured two-dimensional ESPI amplitude of the plate and $\phi(x, y)$ is the measured two-dimensional ESPI phase of the plate. This generated complex plate displacement is then taken as the input to the VEFESPI method in order to determine spatial derivatives and subsequently, vibrational energy flow from the ESPI recorded transverse plate displacement.

\subsection{The effect of noise on vibrational energy flow calculation}

Noise within the ESPI displacement signal is transformed into the wavenumber domain and then amplified when computing the spatial derivatives required for the vibrational energy flow computation. This effect is most pronounced at the high wavenumber locations. To avoid the undesired spectral alteration process the noise contaminated wavenumber spectrum will be filtered by using an ideal 2D filter algorithm as well as an oval 2D Butterworth low-pass filter. Both filter types are defined in the Appendix B. They are applied within the wavenumber domain prior to the $k$-space derivation method.

To determine the optimum filter parameters, optical noise was extracted from the measured ESPI displacement data by transforming the measured infinite plate displacement signal into the wavenumber domain by employing a 2D FFT. Within the wavenumber domain the travelling wave component was deleted. The spectral noise signal was then inverse-transformed and superimposed onto a synthetically generated infinite plate displacement, using the measured force amplitude that was obtained during the ESPI experiment. The following mean squared error function was then used for a range of ascending filter cut-off points. Thus, the total transmitted vibrational power $\left(P_{a}\right)_{F_{\infty}}$ within the infinite plate at each concentric circle of radius $|k r|$ was compared to the vibrational input power $\left(P_{i n}\right)_{F_{\infty}}$ by forming the expression:

$$
\Pi=\frac{1}{N} \sum_{i=1}^{N}\left(1-\frac{\left(P_{a_{i}}\right)_{F_{\infty}}}{\left(P_{i n}\right)_{F_{\infty}}}\right)^{2} .
$$

Here, $N$ is the number of points from each numerical contour integration along $|k r|$. It can be realised that the one-dimensional mean squared error function, Eq. (24), incorporates the two-dimensional energy fields, $\overline{P_{x}}$ and $\overline{P_{y}}$. Thus, the initial two-dimensional optimisation problem is now reduced to a one-dimensional problem, which can be implemented straightforwardly.

Fig. 8a-b shows a measured infinite plate displacement at an excitation frequency of $f_{0}=1503.2 \mathrm{~Hz}$. It is apparent that the displacement data are contaminated by optical noise. Fig. 8c-d shows the extracted ESPI noise. Fig. 9 displays the mean squared error obtained at 50 different filter cut-off points of the $1503.2 \mathrm{~Hz}$ excited infinite plate 
displacement. It can be noticed from Fig. 9a that the optimum cut-off point for the ideal filter is about $7.7 \%$ of the modulus of maximum spectral displacement amplitude. From Fig. 9b it can be seen that the optimum spatial cut-off wavenumber of the Butterworth filter is about $k_{c}=2 k_{r}$.

\section{Experimental results}

\subsection{Point mobility measurement}

Fig. 10 displays the theoretical point mobility computed from Eq. (7) and the measured point mobility computed using the standard $\mathrm{H} 1$ estimator. It can be seen in Fig. 10a that up to $1500 \mathrm{~Hz}$ the measured point mobility follows the trend of the theoretical point mobility. At lower frequencies resonant plate behaviour is still present indicating a lower degree of damping achieved by the anechoic termination and, thus, undesired reflections occur on the plate's boundaries. However, this result is not surprising since the experimental apparatus was designed to effectively dissipate vibrational energy at frequencies greater than $400 \mathrm{~Hz}$. This effect can be seen in Fig. 10b where the theoretical infinite plate point mobility matches well with the measured 'infinite' plate point mobility within the frequency range from $500 \mathrm{~Hz}$ to $1500 \mathrm{~Hz}$. At five selected excitation frequencies, $f_{0}$, experimental "infinite" plate displacements were recorded using the ESPI measurement system. The excitation frequency of $569.7 \mathrm{~Hz}$ was selected as it represented a local minimum of the frequency response function. The excitation frequencies of $605.6 \mathrm{~Hz}$ and 899.5 Hz were selected in order to represent a local maximum of the frequency response function. The remaining two frequencies, at $1194.4 \mathrm{~Hz}$ and $1503.2 \mathrm{~Hz}$, were located close to the theoretical infinite plate response. The excitation frequencies, $f_{0}$, as well as the computed vibrational input power values calculated from both the theoretical impedance based method, Eq. (9), and the measured point mobility based method are shown in Table 2. A comparison of the two calculations of input power for each frequency shows increasing agreement as the frequency increases i.e. as the experimental plate becomes more 'infinite'. However, irrespective of whether the frequency chosen was at a local maximum or a local minimum, all values of input power calculated from the mobility based method are less than the corresponding values from the theoretical impedance based method because of the presence of a small imaginary component in the measured point mobility.

\subsection{Vibrational power measurement and energy balance}

The excitation frequency of $1503.2 \mathrm{~Hz}$ was considered to represent the best compromise between the maximum number of wavelengths within the ESPI window and a measured frequency response function close to the theoretical infinite plate value. Figs. 11a-b display the amplitude and phase of the displacement at $1503.2 \mathrm{~Hz}$, as acquired by the ESPI system. It can be seen in Figs. 11a-b that within the ESPI window some blank (dark colour) regions appear indicating a low laser light illumination. Within these regions, transverse displacement information is missing and the initially recorded amplitude and phase matrices need to be truncated in order to provide the VEFESPI method with useable data. Figs. 11c-d display a top-view of the real and imaginary parts of the 
truncated ESPI displacement. It can be seen in Fig. 11c-d that the displacement is symmetrically truncated around the excitation location. Furthermore, approximately two wavelenghts are included within the truncated ESPI signal. It can be realised from the recorded ESPI displacement, shown in Fig. 11, that a radially symmetric wave is travelling away from the excitation location. Figs. 12a-b display the truncated two-dimensional transverse ESPI displacement. Figs. 12c-d show a cross-section of the transverse ESPI displacement along $y=0$ where the effect of optical noise on the signal can be seen. Fig. 13 displays the complex, 'infinite' plate displacement at $1503.2 \mathrm{~Hz}$, filtered by the ideal 2D filtering process and the oval 2D Butterworth filter using the optimum filter cut-off points established previously. It can be seen in Fig. 13 that compared to the original data shown in Fig. 12a-b that the noise components have been significantly reduced. Both types of filter produced similar results. Fig. 14 shows the filtered active vibrational energy flow maps, as a result of applying the VEFESPI method to the $1503.2 \mathrm{~Hz}$ ESPI measurement. It can be seen in Fig. 14 that the active energy flows radially away from the excitation location, as expected. Both filter types produced similar vibrational energy flow results. The number of data points shown in Fig. 14 was reduced to 16 per side for better visibility of the overall pattern.

As shown in Section 2.3, integration of the active vibrational energy flow around a closed contour is equal to the total transmitted vibrational power through the particular contour $\left(P_{a}\right)_{F_{\infty}}$ and, hence, equal to the vibrational input power injected into the plate. Fig. 15a displays the ESPI measured vibrational energy flow integrated around the excitation location for each location $|k r|$ for both filters. The theoretical impedance based measurement of input power at $1503.2 \mathrm{~Hz}$ is also shown as a horizontal line of value $2.35 \cdot 10^{-4} \mathrm{~W}$. The measured mobility based calculation of input power (not shown) has an almost identical value of $2.31 \cdot 10^{-4} \mathrm{~W}$. In principle, both active energy flow curves should be constant over the $|k r|$ range and equal in value to the input power value. It can be seen in Fig. 15a that the value of the total transmitted power lies close to the measured vibrational input power value for locations where $|k r|>2$. For both of the filtered ESPI measurement curves it can be seen that at locations close to the source, i.e. $|k r|<2$, that the total transmitted power value tends to zero. This is clearly in error since the velocity at the centre of the plate is determined by its characteristic impedance. As noted by Skudrzyk in Ref. [23] a circular disc of radius $|k r|<0.63$ moves with almost the same velocity as the centre point. This problem with the VEFESPI total transmitted power calculation may be due to the numerical integration process as the error becomes more significant as fewer points are included in the integration process. However, since the VEFESPI method is only applicable to the vibrational farfield and this error occurs outside the intended $k r$ range of the VEFESPI method the error will not be considered further. At locations towards the edge of the ESPI window, where $|k r|>11$, the total transmitted power curves start to diverge from the input power measurement. This may be due to the curve wrap method or the filtering applied in the wavenumber domain. Fig. 15b displays the relative difference $\Delta \varepsilon$ between the ESPI measured total transmitted power $\left(P_{a}\right)_{F_{\infty}}$ and the theoretical impedance based measurement of input power $\left(P_{i n}\right)_{F_{\infty}}$, computed as: 


$$
\Delta \varepsilon=\left(1-\left(P_{a}\right)_{F_{\infty}} /\left(P_{i n}\right)_{F_{\infty}}\right) \cdot 100 \%
$$

It can be seen in Fig. 15b that the relative difference between the input power and the transmitted power is less than $\pm 20 \%(<1 \mathrm{~dB})$ for most regions of the plate.

Fig. 16 displays the truncated ESPI measured plate displacement at $1194.4 \mathrm{~Hz}$. Fig. 17 displays the filtered active vibrational energy flow maps and Fig. 18 displays a comparison of the ESPI measured total transmitted power with the theoretical impedance based measurement of vibrational input power at $1194.4 \mathrm{~Hz}$. The impedance based measurement of input power is shown as a horizontal line at $2.45 \cdot 10^{-4} \mathrm{~W}$. The measured mobility based calculation of input power (not shown) has a $6.5 \%$ lower value of $2.29 \cdot 10^{-4}$ W. It can be realised from Fig. 18 that at $1194.4 \mathrm{~Hz}$ a good agreement between the total transmitted vibrational power and the input power was achieved, with differences again being in a range of $\pm 20 \%(<1 \mathrm{~dB})$.

Figs. 19-21 display the corresponding results from the 899.5 Hz measurement. From the ESPI amplitude and phase maps shown in Figs. 11, 16 and 19, it can be realised that the experimental "infinite" plate rig increased its anechoic efficiency with increasing frequency. At the higher frequencies, infinite plate behaviour could be simulated much better and, thus, at the excitation frequencies of $899.5 \mathrm{~Hz}, 1194.4 \mathrm{~Hz}$ and $1503.2 \mathrm{~Hz}$ a good "infinite" plate response was recorded. This infinite plate behaviour is also apparent in the active vibrational energy flow maps at $1194.4 \mathrm{~Hz}$ and $899.5 \mathrm{~Hz}$ shown in Figs. 17 and 20, respectively. Fig. 21 displays a comparison of the ESPI measured total transmitted power with the theoretical impedance based measurement of vibrational input power at $899.5 \mathrm{~Hz}$. The impedance based measurement of input power is shown as a horizontal line at $1.73 \cdot 10^{-4}$ W. The measured mobility based calculation of input power (not shown) has a $12 \%$ lower value of $1.52 \cdot 10^{-4} \mathrm{~W}$. It can be realised from Fig. 21 that at $899.5 \mathrm{~Hz}$, a good agreement between the total transmitted vibrational power and the input power was achieved, with differences being in a range of $\pm 20-25 \%$, respectively, depending upon whether the theoretical impedance based or experimental mobility based measurement of input power is used for comparison.

Fig. 22 displays the truncated ESPI measured plate displacement at $605.6 \mathrm{~Hz}$. Fig. 23 displays the filtered active vibrational energy flow maps and Fig. 24 displays a comparison of the measured total transmitted vibrational power with the measured vibrational input power at $605.6 \mathrm{~Hz}$. Figs. 25-27 display the corresponding results from the $569.7 \mathrm{~Hz}$ measurement. From the ESPI displacement maps shown in Figs. 22 and 25, measured at the excitation frequencies of $569.7 \mathrm{~Hz}$ and $605.6 \mathrm{~Hz}$, respectively, it is apparent that some wave reflections from the embedded plate's edge occurred and, thus, a standing wave environment, to some extent, was present. It can be noticed from the active energy flow maps shown in Figs. 23 and 26 that the active energy flows away from the excitation location. However, at some angles dominant energy flow paths are also apparent indicating that vibrational energy no longer travels in a radial symmetrical manner away from the source. Thus, some of the initial outgoing radial wave energy is reflected at the plate's boundaries. This effect is also apparent in Figs. 24 and 27 which compare the total transmitted vibrational power with the theoretical impedance based measurement of input power at $605.6 \mathrm{~Hz}$ and $569.7 \mathrm{~Hz}$, respectively. At these two frequencies differences of up to $60 \%$ and $90 \%$, respectively, can be seen between the total transmitted vibrational power 
and the theoretical impedance based input power measurement over the wavenumber range where $k r>1$. These differences are even greater, $80 \%$ and $105 \%$, respectively, if the experimental mobility based measurement of input power is used. This is not surprising since Eq. (6) is true only for purely infinite wave motion. If reflections on the plate boundaries need to be taken into account then vibrational input power supplied by an external source is equal to the transmitted vibrational power moving out of the volume through the volume boundaries plus the vibrational power dissipated within the volume. For purely infinite structures the dissipation process may often be neglected, however for finite structures not.

\section{Summary and conclusions}

This paper has presented a theoretical study and an experimental investigation of vibrational energy flow in a transverse point force excited infinite plate. Time-averaged vibrational power expressions, Eqs. (1)-(4), were presented based on the spatial partial derivatives of the plate's displacement field. From this starting point, and in a novel approach, a theoretical analysis of vibrational energy flow in an infinite plate was undertaken by introducing a simplification to the plate's displacement expression, Eq. (11), based upon the truncated asymptotic expansion of the Hankel function difference. This approximation restricted the verification of the vibrational energy flow analysis to locations in the plate where the product $|k r|$ was greater than 4 . However, this approximation was considered acceptable as this study is primarily interested in the active vibrational energy flow in the farfield of an infinite plate.

Since the wave propagation within an infinite plate is of a radially symmetric nature, a polar active vibrational energy flow per unit length expression, Eqs. (12) was derived. The total transmitted vibrational power, Eq. (15), was obtained by integrating the active vibrational energy flow per unit length over a closed circular contour. From a comparison with the vibrational input power, an error term in the derived total transmitted vibrational energy flow, Eq. (15), could be identified. This error term is caused by using the truncated asymptotic expansion of the Hankel function difference. When computing total transmitted vibrational energy by using a square contour integration, Eq. (18), a slightly reduced error term was identified.

To assess the accuracy of the VEFESPI method, vibrational energy flow quantities were produced using synthetically generated 'infinite' plate displacement data. However, the computation of vibrational energy flow suffered from signal leakage due to the analysis of a radially symmetric displacement signal that was truncated to a rectangular Cartesian coordinate based displacement matrix. This undesired signal leakage was partly corrected by using the curve wrap technique.

Active vibrational energy flow within the plate structure was determined from two complex-valued vibrational power per unit length fields denoted as $\overline{P_{x}}$ and $\overline{P_{y}}$. With this information it was possible to construct active vibrational energy flow maps exhibiting the magnitude and direction of active vibrational energy flow within the plate. A twodimensional relative error distribution, Eq. (24), was calculated by comparing the determined VEFESPI active energy flow to the theoretical value of the active vibrational 
energy flow in the $x$ and $y$ directions, as given by Eq. (17). The relative error for the active vibrational energy flow was shown to be less than $0.5 \%$.

Energy balance comparisons within the infinite plate were carried out by integrating the active vibrational energy flow fields in the $x$ and $y$ directions along a closed square contour that was located around the excitation location. This integration process produced the total transmitted vibrational power. By comparing this quantity to the vibrational input power an assessment was made, via Eq. (24), of the error due to the VEFESPI energy flow computation. This procedure had the advantage that the two-dimensional vibrational power fields, $\overline{P_{x}}$ and $\overline{P_{y}}$, could be incorporated into a one-dimensional mean square error function, as given by Eq. (24). It was shown that at Helmholtz numbers greater than $|k r| \approx 5$ the total transmitted vibrational power within the infinite plate is equal to the input power. At values of $|k r|<5$ the trend follows that expected by the error terms identified in Eqs. (15) and (18).

Vibrational energy flow was also computed from measured 'infinite' plate displacements, acquired by an ESPI measurement system. From the measured point mobility of the plate five specific excitation frequencies within the frequency range $570 \mathrm{~Hz}$ to $1503 \mathrm{~Hz}$ were selected for analysis. For measured ESPI data it is known that the accuracy of vibrational energy flow computation will be reduced because of the presence of noise in the wavenumber domain during the $k$-space derivation process [18]. Thus, wavenumber domain filtering was carried out by applying an ideal $2 \mathrm{D}$ filtering procedure as well as the oval 2D Butterworth filter. Optimised filter cut-off points were determined by superimposing ESPI noise onto synthetic displacement data and then evaluating the mean square error function, Eq. (24). The ideal 2D filter and the oval 2D Butterworth filter both yielded similar vibrational energy flow results.

To assess the accuracy of the ESPI measured vibrational energy flow, the active vibrational energy flow maps were integrated along a closed contour around the excitation location. The closed contour integration resulted in the total transmitted vibrational power through that particular contour. A comparison between the ESPI based total transmitted vibrational power and the measured vibrational input power showed good agreement between both measures with differences being in a range of $\pm 20-25 \%$ for the three highest frequencies tested. Larger differences were apparent at the two lowest frequencies analysed. This was attributed to wave reflections from the plate's boundaries. Nevertheless, active vibrational energy flow maps from "infinite" plate displacements that include some standing waves, as present at the $569.7 \mathrm{~Hz}$ and $605.6 \mathrm{~Hz}$ measurements, could be constructed. However, simple energy balances, as carried out by evaluating Eq. (6) were not appropriate anymore in order to assess any difference between the total transmitted vibrational power and the power injected into the plate.

However, a number of challenges remain to the application of the VEFESPI system to 'real world' structures. For example, due to a number of blank regions present within the acquired ESPI amplitude and phase maps, the usable spatial range of the measured plate displacement was restricted. This restriction was forced by truncating the data around the off-centre excitation location. For example, at the $1503.2 \mathrm{~Hz}$ measurement only two wavelengths of displacement data could be included when employing the spatial Fourier transform. Furthermore, a knowledge of the true spatial resolution, or spatial sampling interval, employed during the ESPI experiment measurement is crucial for 
accurate vibrational energy flow calculation. It was shown in Ref. [18] that a relative change in the spatial resolution of the ESPI system results in a linear change in the measured ESPI vibrational energy flow. For this research, the spatial resolution, $\Delta x$, of the ESPI measurements was determined from the length of the ESPI window and the number of data points included within the window, i.e. $\Delta x=L_{x} / N_{x}$.

In conclusion, energy flow in the vibrational farfield $(k r>4)$ of an 'infinite' plate that contains radially symmetric bending wave propagation can be measured by the VEFESPI method to a good degree of accuracy $( \pm 20-25 \%)$ if appropriate filtering to reduce the effects of data truncation and optical noise are applied.

\section{Acknowledgement}

The authors would like to thank Mr. M. Dale for his assistance with the ESPI measurements.

\section{References}

1. D. U. Noiseux, Measurement of power flow in uniform beams and plates, Journal of the Acoustical Society of America 47 (1970) 238-243.

2. G. Pavic, Measurement of structural borne wave intensity, part I: Formulation of the methods, Journal of Sound and Vibration 49 (1976) 221-230.

3. J. W. Verheij, Cross spectral density methods for measuring structure borne power flow in beams and pipes, Journal of Sound and Vibration 70 (1980) 133-139.

4. W. Redman-White, The experimental measurement of flexural wave power flow in structures, $2^{\text {nd }}$ International Conference on Recent Advances in Structural Dynamics, 1984, pp. 467-474.

5. E. G. Williams, H. D. Dardy, R. G. Fink, A technique for measurement of structure-borne intensity in plates, Journal of the Acoustical Society of America 78 (1985) 2061-2068.

6. J. D. Maynard, E. G. Williams, Y. Lee, Nearfield acoustic holography: I. theory of generalized holography and the development of NAH, Journal of the Acoustical Society of America 78 (1985) 1395-1413.

7. R. J. Unglenieks, R. J. Bernhard, Measurement of flexural structural intensity using wavenumber techniques, $4^{\text {th }}$ International Congress on Intensity Techniques, 1993, pp. 305-311.

8. R. Morikawa, S. Ueha, K. Nakamura, Error evaluation of the structural intensity measured with a scanning laser Doppler vibrometer and a K-Space signal processing, Journal of the Acoustical Society of America 99 (1996) 2913-2921.

9. A. Nejade, R. Singh, Flexural intensity measurements on finite plates using modal spectrum ideal filtering, Journal of Sound and Vibration 256 (2002) 33-63.

10. J.-C. Pascal, X. Carniel, V. Chalvidan, P. Smigielski, Energy flow measurements in high standing vibration fields by holographic interferometry, Inter Noise 95, 1995, pp. 625-630. 
11. J.-C. Pascal, X. Carniel, V. Chalvidan, P. Smigielski, Determination of phase and magnitude of vibration for energy flow measurements in a plate using holographic interferometry, Optics and Lasers in Engineering 25 (1996) 343-360.

12. J.-C. Pascal, J.F. Li, X. Carniel, Wavenumber Processing Techniques to Determine Structural Intensity and Its Divergence from Optical Measurements without Leakage Effects, Shock and Vibration 9(1) 2002 57-66.

13. P. S. Alves, J. R. Arruda, L. Gaul, S. Hurlebaus, Power flow estimation using pulse ESPI, $4^{\text {th }}$ International Conference on Vibration Measurements by Laser Techniques: Advances and Applications, 2000, pp. 362-373.

14. J. R. Arruda, Surface smoothing and partial spatial derivatives computation using a regressive discrete Fourier series, Mechanical Systems and Signal Processing 6 (1992) 41-50.

15. J. P. Chambard, V. Chalvidan, X. Carniel, J.-C. Pascal, Pulsed TV-holography recording for vibration analysis applications, Optics and Lasers in Engineering 38 (2002) 131-143.

16. X. Carniel, J.-C. Pascal, J. F. Li, Structural intensity and acoustic radiation from optical measurements: The VIPART software, Proceedings - SPIE The International Society For Optical Engineering, 2000, pp. 258-265.

17. T. Eck, S. J. Walsh, M. Dale, N. C. Taylor, Vibrational power flow measurements in a beam using electronic speckle pattern interferometry, Acta Acustica United with Acustica, 92 (2006) 765-776.

18. T. Eck, Measurement of vibrational energy flow using electronic speckle pattern interferometry, Ph.D. Thesis, Loughborough University, 2008.

19.H.G. Goyder, R.G. White, Vibrational power flow from machines into built-up structures, part I: Introduction and approximate analyses of beam and plate-like foundations, Journal of Sound and Vibration 68 (1980) 59-75.

20. R. Lande, R.S. Langley, The energetics of cylindrical bending waves in a thin plate, Journal of Sound and Vibration 279 (2005) 513-518.

21. L. Cremer, M. Heckl, B.A.T. Petersson, Structure-borne sound: Structural vibrations and sound radiation at audio frequencies, $3^{\text {rd }}$ edition, Springer Verlag, Berlin Heidelberg, 2005.

22. W.X. Li, L.D. Mitchell, M.L. Neumann, Mapping nonsquare and unevenly spaced 2D SLDV data of an aircraft fuselage by using spatial DFT-IDFT techniques, Shock and vibration, 3 (1996) 135-140.

23. E. Skudrzyk. Simple and complex vibratory systems. Pennsylvania State University Press, 1968.

\section{Appendix A: The curve wrap technique}

In practice ESPI data are often recorded with a non-integer number of wavelengths. Since the VEFESPI method employs a spatial Fourier transform, a periodic set of data is essential otherwise signal leakage will falsify the computation of the spatial derivatives 
and, hence, the accuracy of the computed vibrational energy flow. Within this work three techniques are investigated that deal with the difficulties arising from non-periodic data.

Two techniques published in the literature utilise different mirroring and reversing techniques of the original data in order to obtain a periodic data sequence. Li et al. propose a periodisation technique, which mirrors reverses and shears the original nonperiodic data [22]. This method is addressed in this work as the mirror-reverse-shear (MRS) periodisation technique. A second periodisation technique was proposed by Pascal et al. [12]. This method will be addressed in this work as the mirror-processing (MP) technique. In the MP procedure the original set of data is mirrored about the $x$-axis and truncated by half. The half-sized mirror signals are then connected to both ends of the original signal. The third technique, proposed by the authors, simply manipulates the vibrational energy flow data to obtain the desired result and is referred to as the curve wrap $(\mathrm{CW})$ technique.

To illustrate the effect of the different techniques a complex, point force excited infinite beam displacement is generated which is non-periodic, with respect to the wavelength. The ESPI data is assumed to be recorded over a $1 \mathrm{~m}$ long section of the beam's length. Fig. A1a displays the resulting transmitted power calculation from the VEFESPI method together with the corresponding input power value of a point excited infinite beam calculated using the standard theoretical formula [21]. It can be seen from Fig. A1a that the VEFESPI transmitted power curve oscillates around the input power value. This oscillation is a consequence of the multiplication process in wavenumber domain in order to obtain the desired spatial derivatives. It can be realised from Fig. A1a that two wrapping curves can be constructed, which enclose the oscillating transmitted power (see black dotted curves). One curve includes all the odd-numbered VEFESPI samples and the other includes all the even-numbered VEFESPI samples. Thus, the sample size of both curves is half the sample size of the original VEFESPI curve. If one interpolates both curves in order to obtain the original sample size of the VEFESPI curve and calculates the mean of both curves, an approximate transmitted power, $\left(P_{x}\right)_{a p p}$ can be found simply as:

$$
\left(P_{x}\right)_{a p p}=\frac{1}{2}\left(\left(\widetilde{P_{x}}\right)_{o d d}+\left(\widetilde{P_{x}}\right)_{e v e n}\right)
$$

Herein, $\left(\widetilde{P_{x}}\right)_{o d d}$ is the interpolated odd-numbered curve wrap and $\left(\widetilde{P_{x}}\right)_{\text {even }}$ is the interpolated even-numbered curve wrap. This method can be used as a simple approximation of the oscillating transmitted power curves due to signal irregularities.

All three techniques were applied with the VEFESPI method and the resulting VEFESPI determined transmitted power curves are compared to the input power data in Fig. A1b. It can be seen in Fig. A1b that there is still some oscillation of the transmitted power at both ends of the ESPI signal length. However, in a range from $0.1 \mathrm{~m}<x<0.9 \mathrm{~m}$ all three techniques achieve good results. Due to the simplicity of its application, the CW method was selected for implementation on the infinite plate.

\section{Appendix B: the 2D Ideal filter and the 2D Butterworth filter}


Within this work the ideal filter is implemented such that the noise contaminated wavenumber spectrum $U\left(k_{x}, k_{y}\right)$ is searched element by element for components that are smaller than or equal to the product of a selected constant $C$ and the maximum magnitude of the wavenumber spectrum. If this condition is satisfied, the particular wavenumber spectrum component is simply set to zero. Thus, the ideal filter process acts as a multiplication of the noise contaminated wavenumber spectrum with zeros and ones at specifically defined positions. This filter can be implemented as:

$$
F\left(k_{x}, k_{y}\right)=\left\{\begin{array}{cc}
1 & \text { for }\left|U\left(k_{x}, k_{y}\right)\right|>C\left|U\left(k_{x}, k_{y}\right)\right|_{\max } \\
0 & \text { for }\left|U\left(k_{x}, k_{y}\right)\right|<C\left|U\left(k_{x}, k_{y}\right)\right|_{\max }
\end{array}\right\} .
$$

Herein, $C$ is a real-valued constant between $0 \leq C \leq 1$. The advantage of this method is that it is straightforward to implement on different programming platforms. The optimum cut-off point can be found by running an automated trial and error computation to find the optimum filter parameter $C$ that removes a significant amount of noise and yet retains a sufficient amount of signal information.

An alternative filter type to the ideal filter is a low-pass 2D Butterworth filter. The oval Butterworth filter shape is given by:

$$
F\left(k_{x}, k_{y}\right)=\frac{1}{1+\left(\frac{r}{K_{c}}\right)^{2 n}}=\frac{1}{1+\left(\left(\frac{X}{K_{c_{x}}}\right)^{2}+\left(\frac{Y}{K_{c_{y}}}\right)^{2}\right)^{2 n}}
$$

It can be seen by Eq. (B2) that the oval Butterworth filter is the magnitude of the wellknown two-dimensional Butterworth filter. The coordinates $X$ and $Y$ are the filter lengths in the $K_{x}$ and $K_{y}$ directions, respectively, and $n$ is the order of the filter that changes the filter slope at the cut-off wavenumbers $k_{c_{x}}$ and $k_{c_{y}}$. 


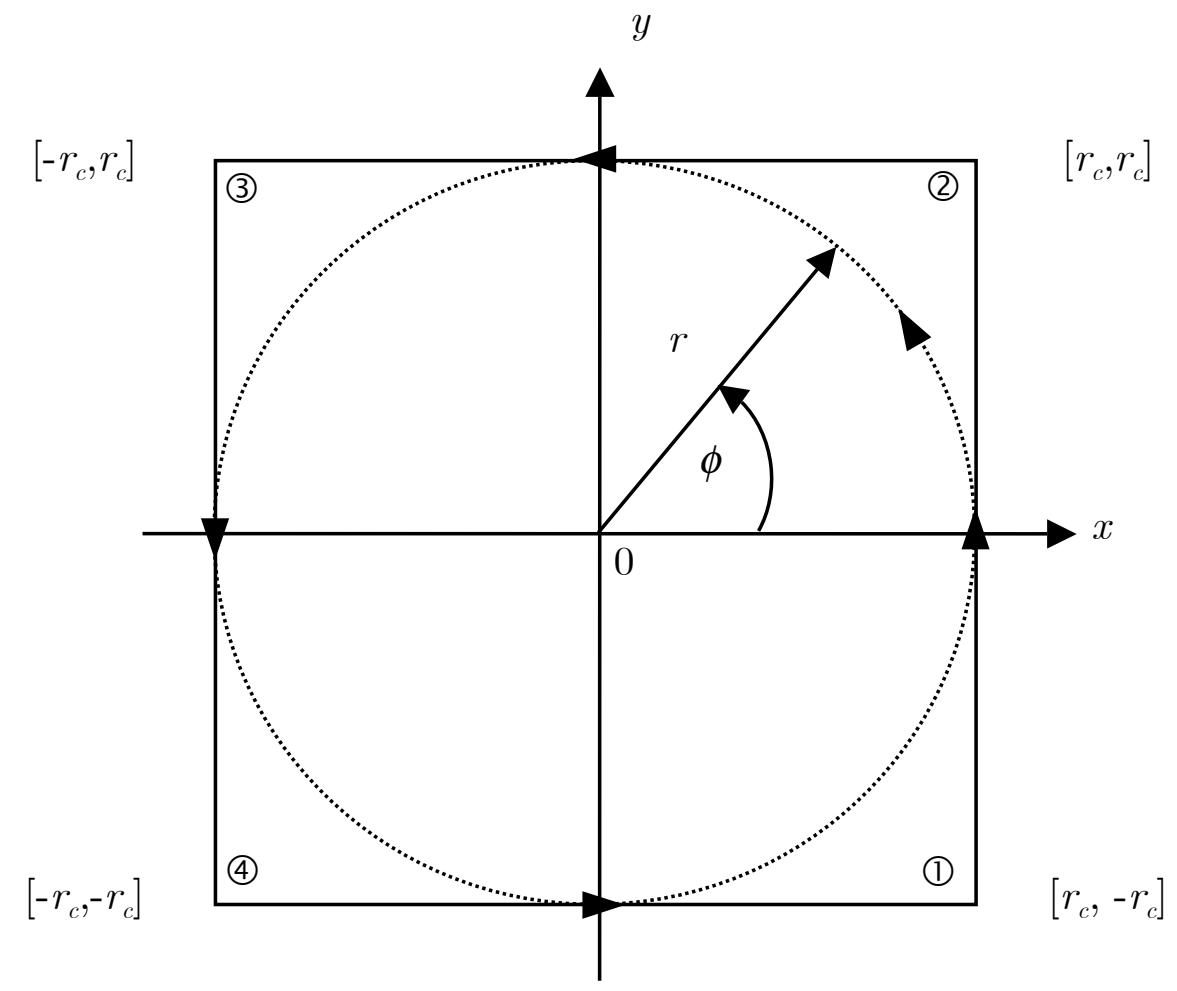

Fig. 1. Two integration contours in the vibrational energy flow field of an infinite plate. 


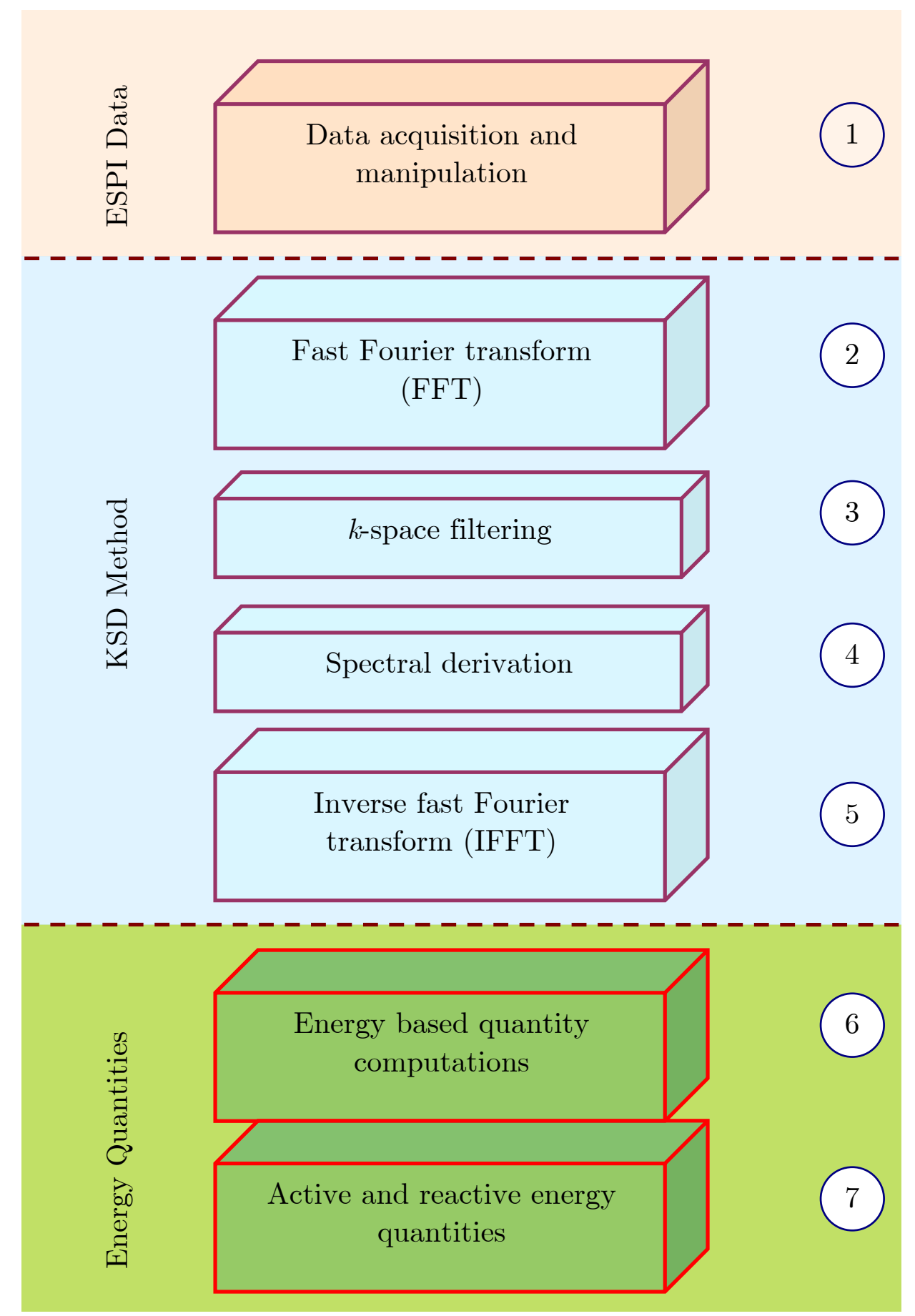

Fig. 2. Flow diagram of the VEFESPI method. 


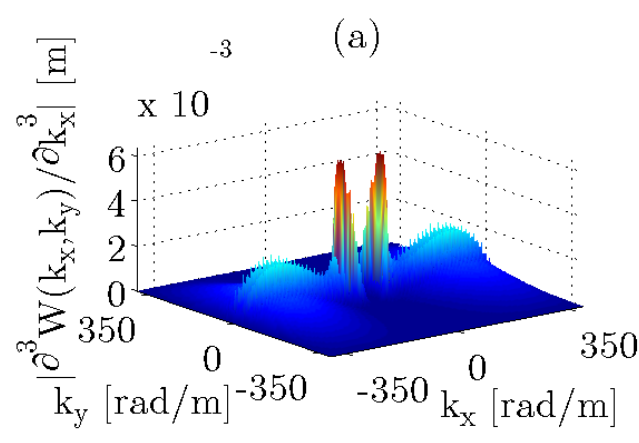

(b)

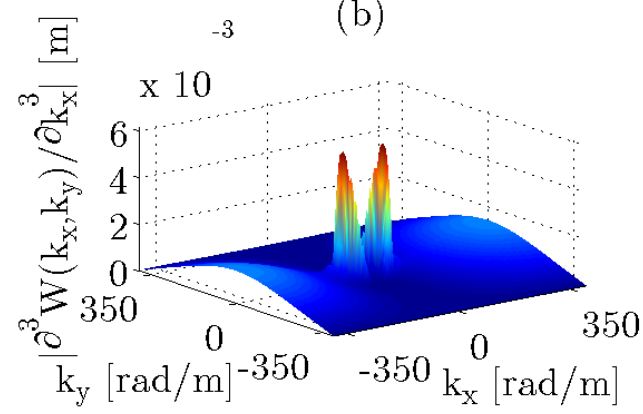

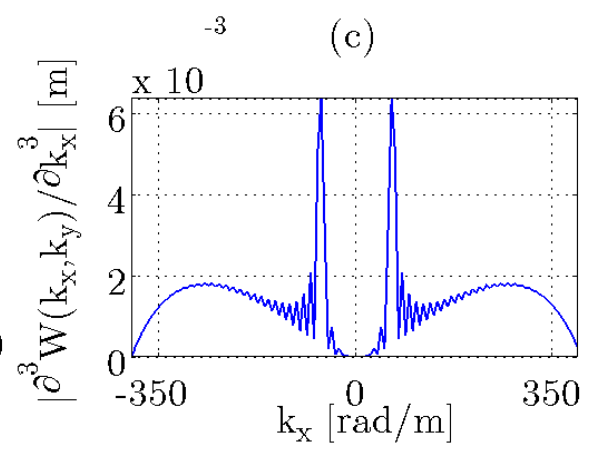

$-3 \quad(d)$

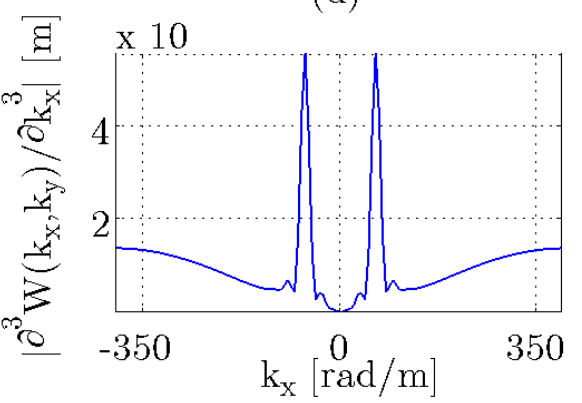

Fig. 3. Comparison of the VEFESPI determined and the theoretical solution of the $3^{\text {rd }}$ spatial derivative with respect to $k_{x}$ of the infinite plate displacement at $f_{0}=986.7 \mathrm{~Hz}$ : (a) 2D modulus VEFESPI; (b) 2D modulus - exact solution; (c) 1D modulus at $k_{y}=0$ - VEFESPI; (d) $1 \mathrm{D}$ modulus at $k_{y}=0$ - exact solution. 


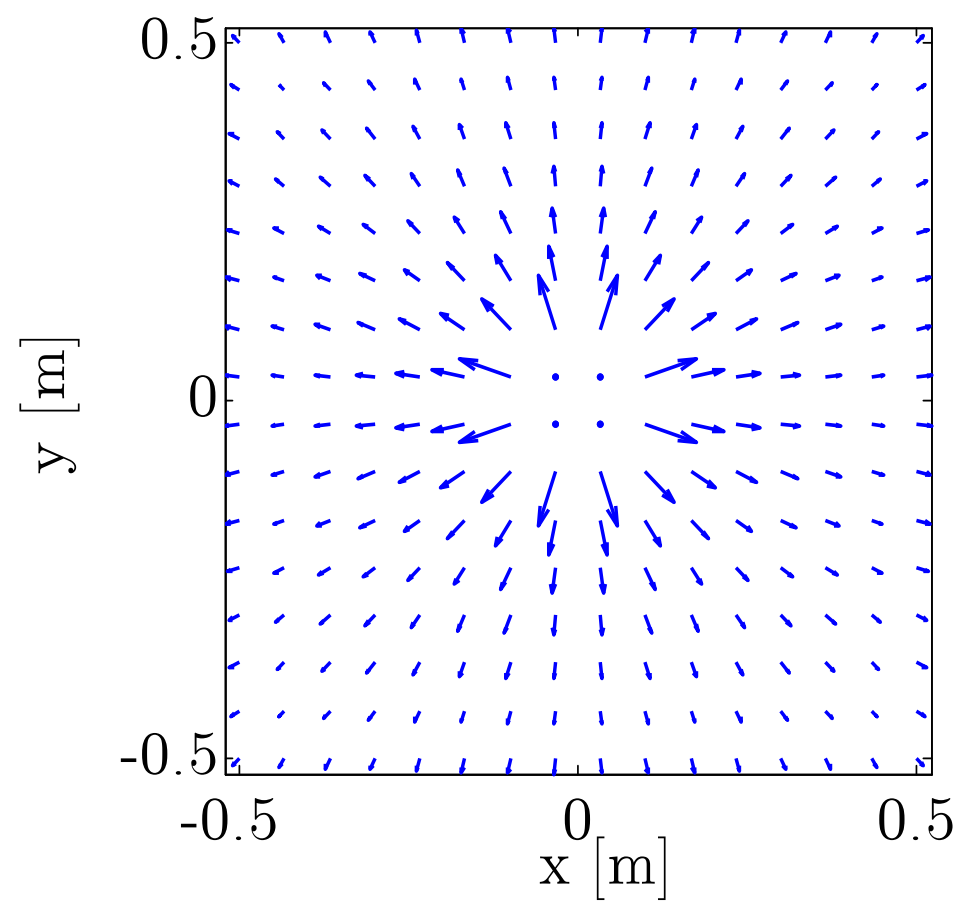

Fig. 4. Active vibrational energy flow map of the infinite plate displacement at $f_{0}=986.7 \mathrm{~Hz}$ from the VEFESPI + curve wrap method. Magnitude of the maximum energy flow vector shown represents approximately $3 \cdot 10^{-3} \mathrm{~W} / \mathrm{m}$. 
(a)

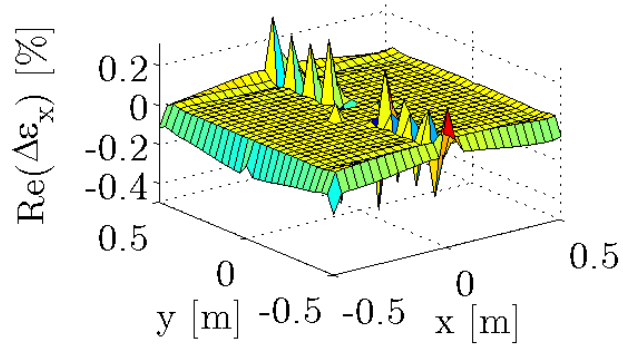

(b)

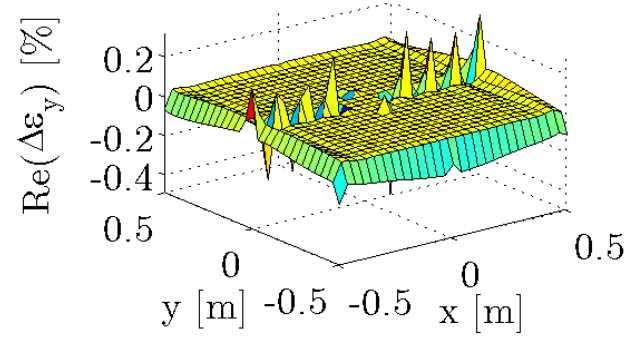

Fig. 5. Relative error of active vibrational energy flow of the infinite plate displacement at $f_{0}$ $=986.7 \mathrm{~Hz}$ : (a) $x$ direction; (b) $y$ direction. 


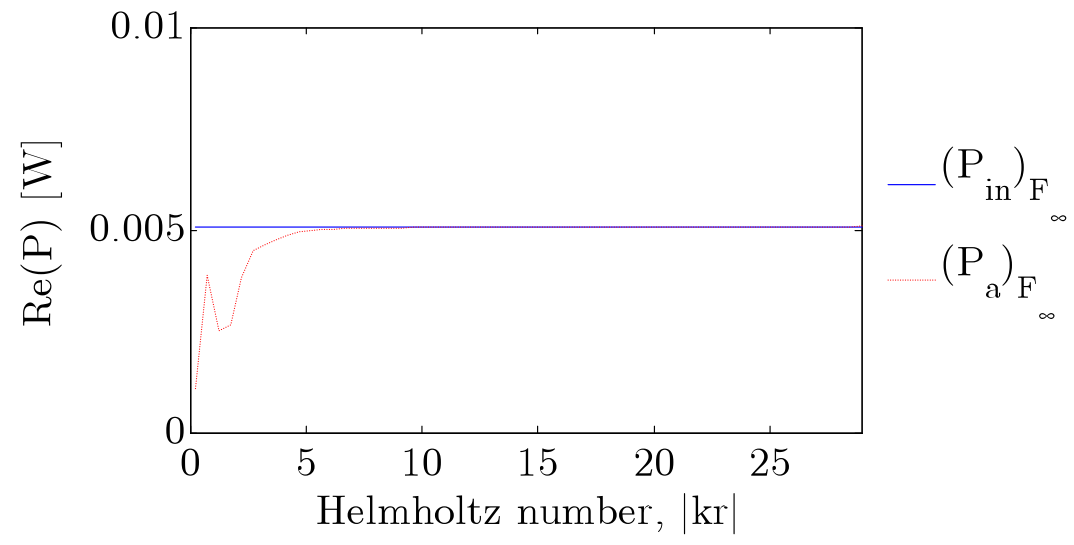

Fig. 6. Comparison of total transmitted vibrational power with the vibrational input power of the infinite plate displacement at $f_{0}=986.7 \mathrm{~Hz}$. 


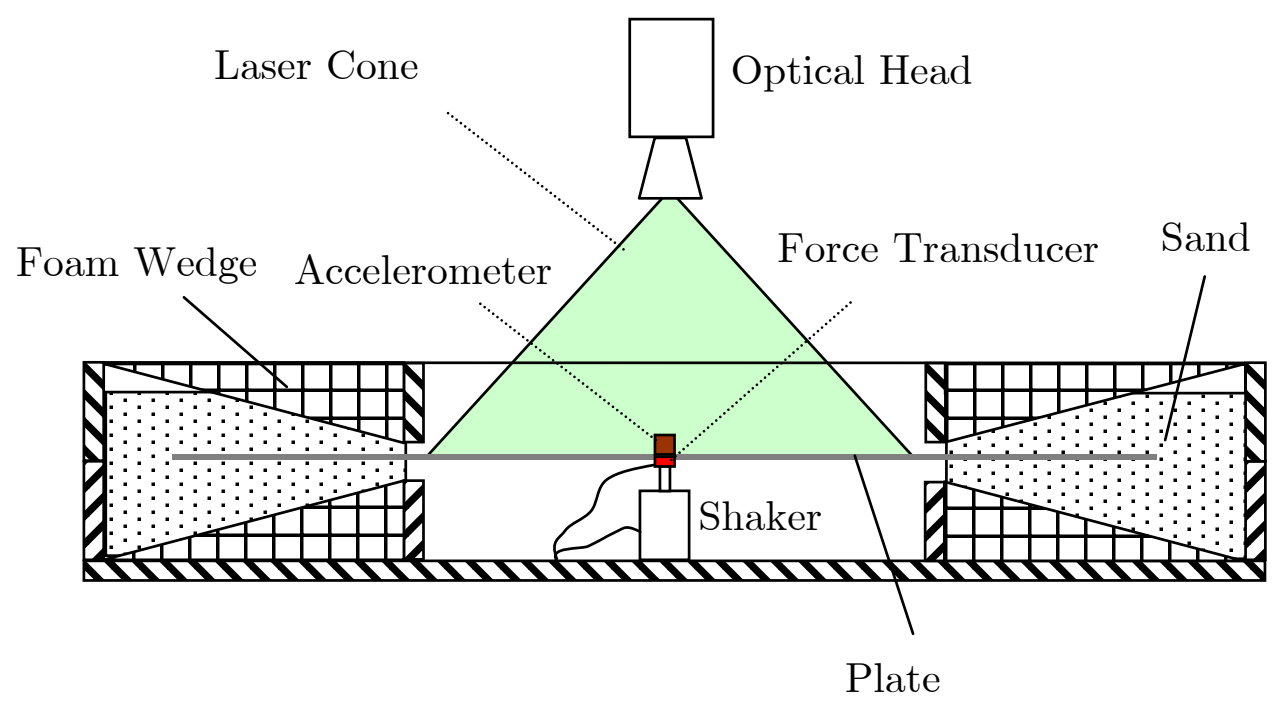

Fig. 7. Cross-sectional view of the force excited experimental "infinite" plate apparatus. 
(a)

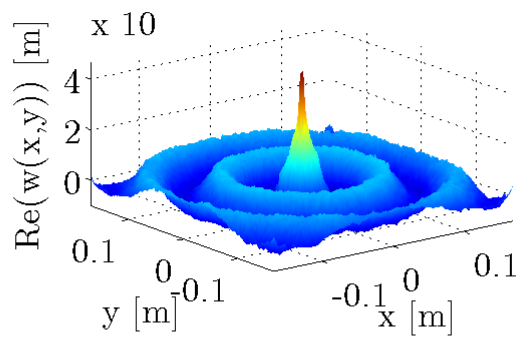

(b)

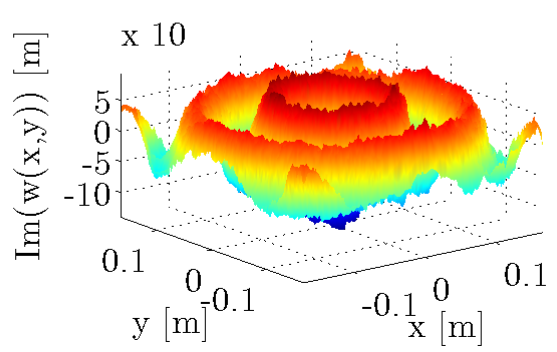

(c)

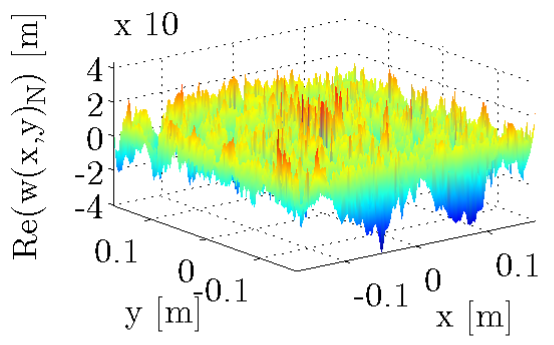

(d)

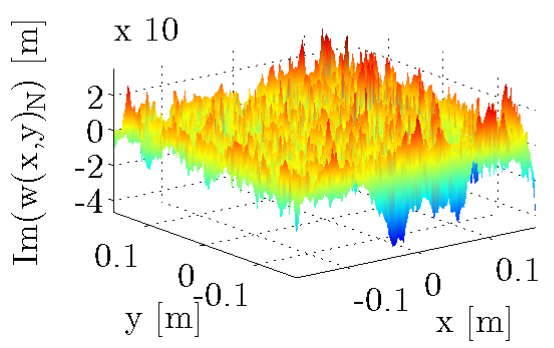

Fig. 8. Noise contaminated infinite plate displacement and extracted ESPI noise at $1503.2 \mathrm{~Hz}$ : (a) 2D real displacement part; (b) 2D imaginary displacement part; (c) 2D real noise part; (d) 2D imaginary noise part. 
(a)

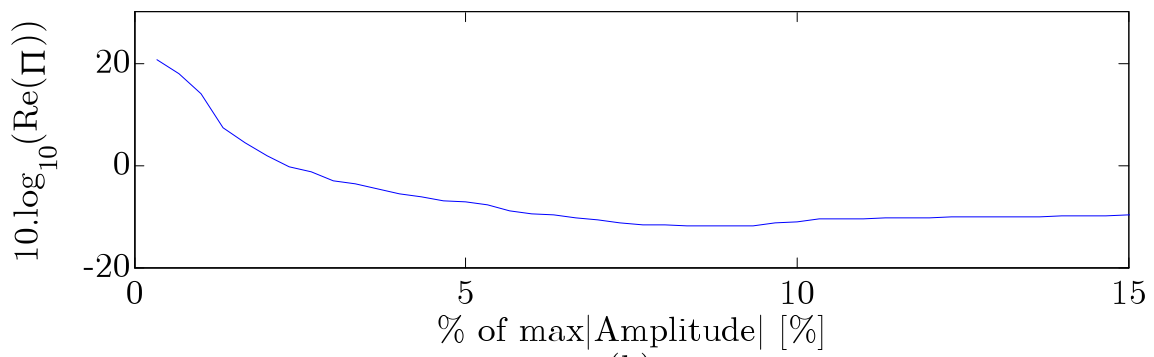

(b)

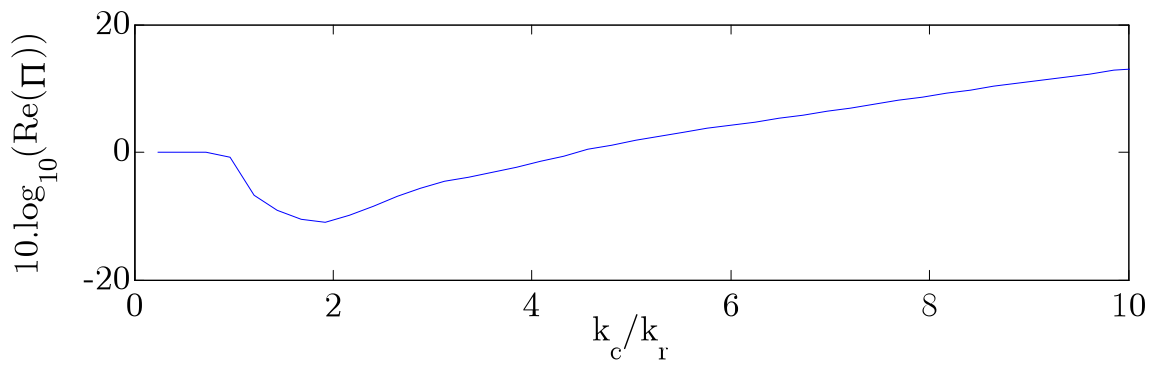

Fig. 9. Relative mean square error of total transmitted power of the infinite plate displacement at 1503.2 Hz: (a) ideal filter; (b) Butterworth filter. 
(a)

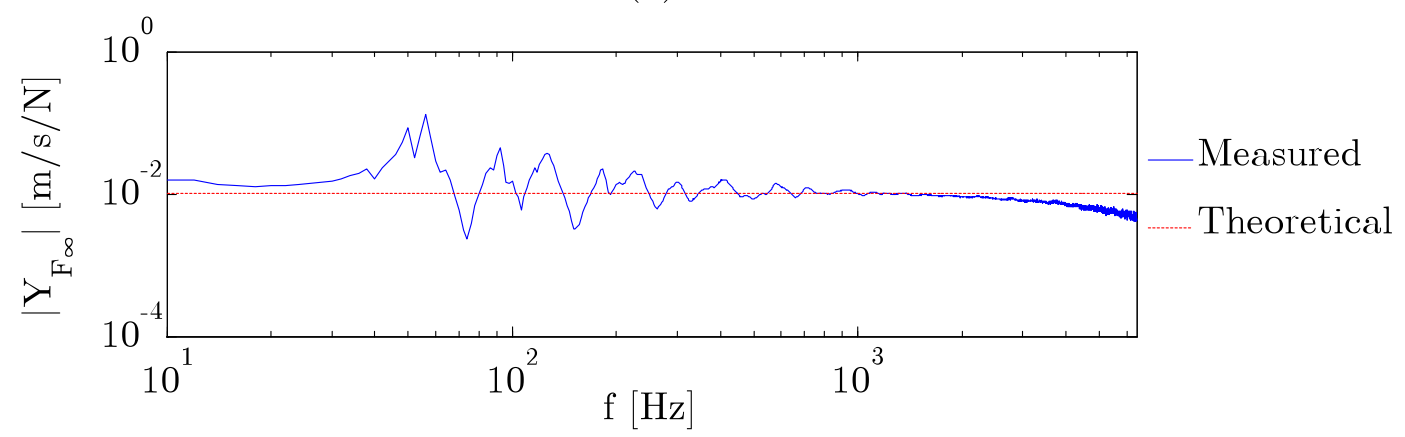

(b)

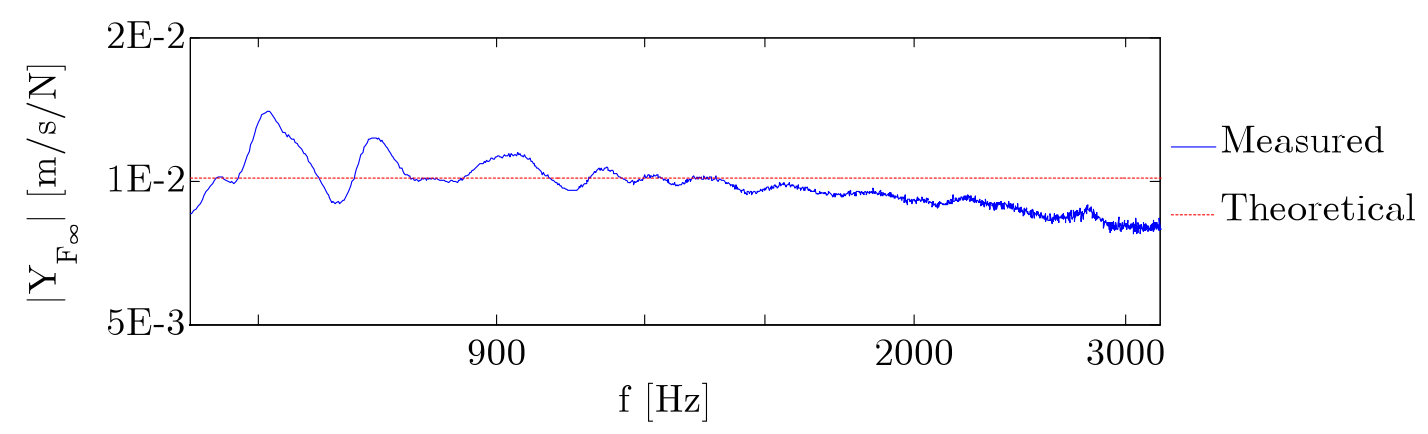

Fig. 10. Modulus of the measured and theoretical point mobility of the force excited "infinite" ESPI plate: (a) $10 \mathrm{~Hz}-6.4 \mathrm{kHz}$; (b) 500-3200 Hz. 
(a)

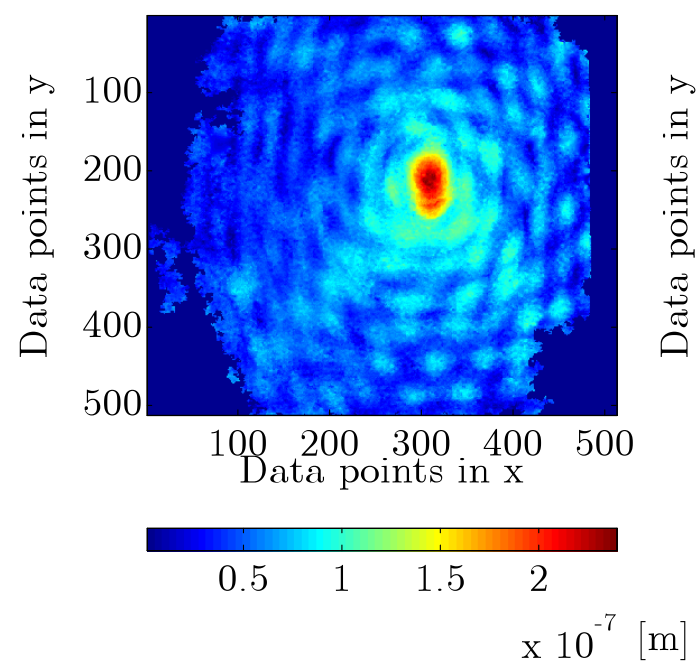

(b)

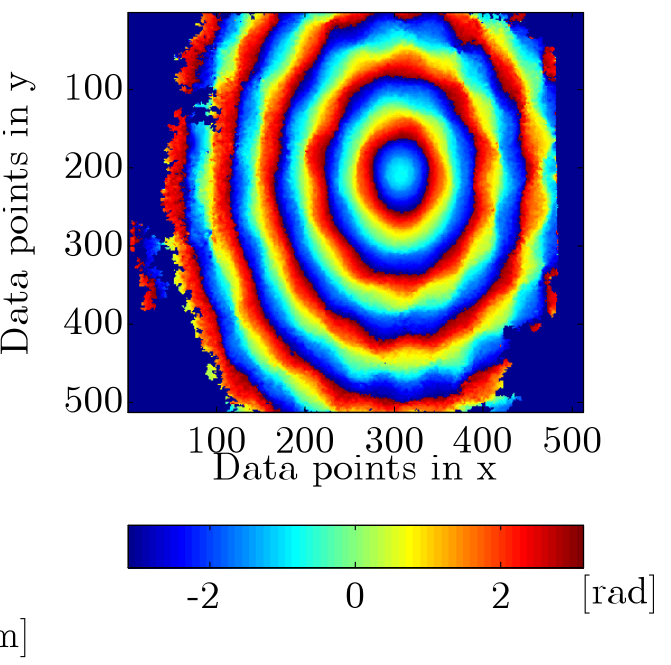

(d)

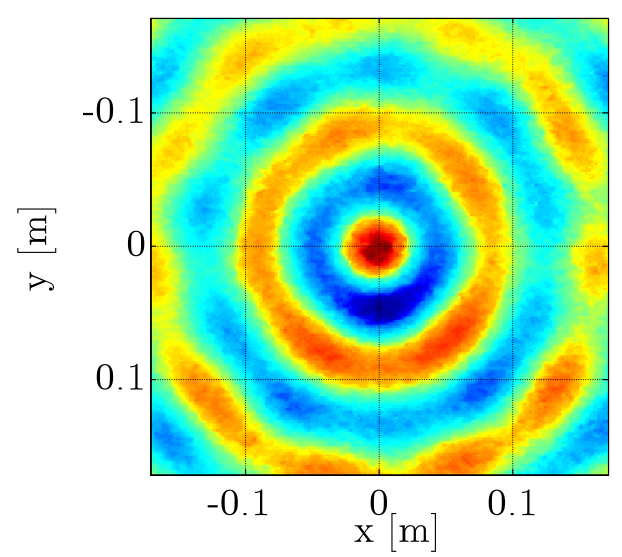

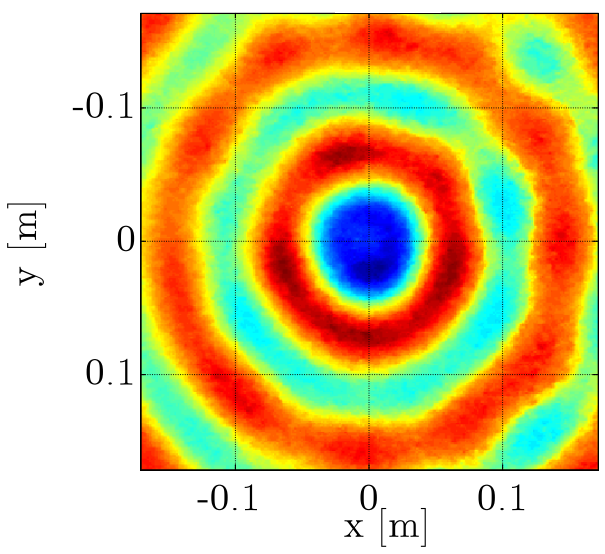

Fig. 11. Top view ESPI image of the measured "infinite" plate displacement at $1503.2 \mathrm{~Hz}$ : (a) amplitude; (b) phase; (c) truncated real part; (d) truncated imaginary part. 
(a)

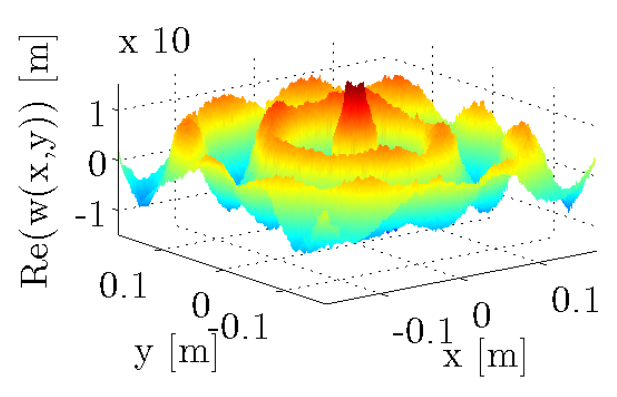

(b)

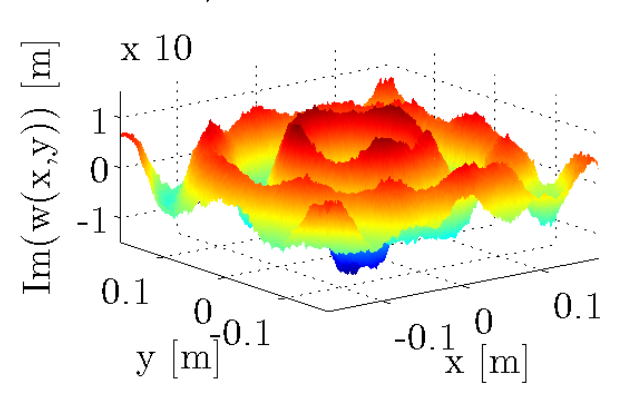

(c)

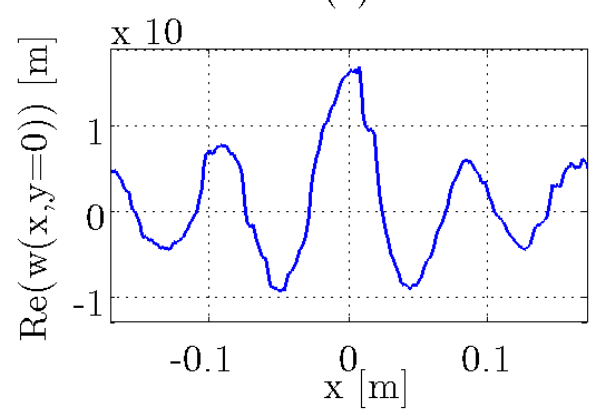

(d)

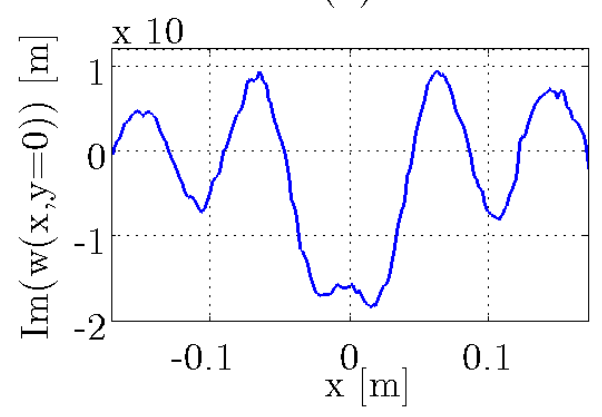

Fig. 12. ESPI image of the measured and truncated "infinite" plate displacement at $1503.2 \mathrm{~Hz}$ : (a) $2 \mathrm{D}$ real part; (b) 2D imaginary part; (c) real part at $y=0$; (d) imaginary part at $y=0$. 
(a)

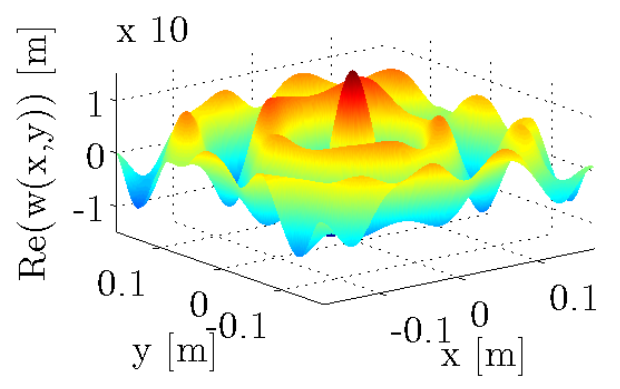

(b)

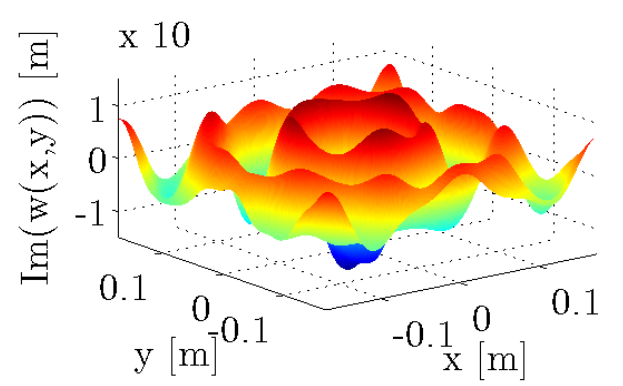

(c)

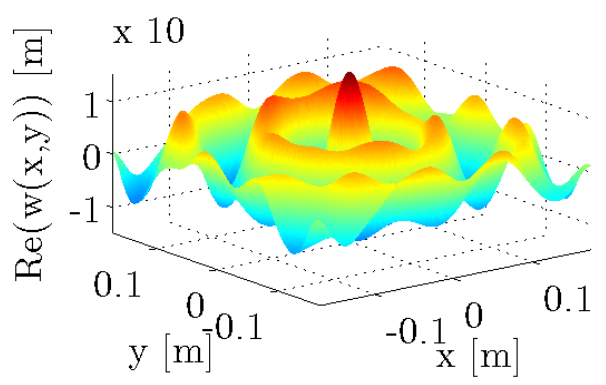

(d)

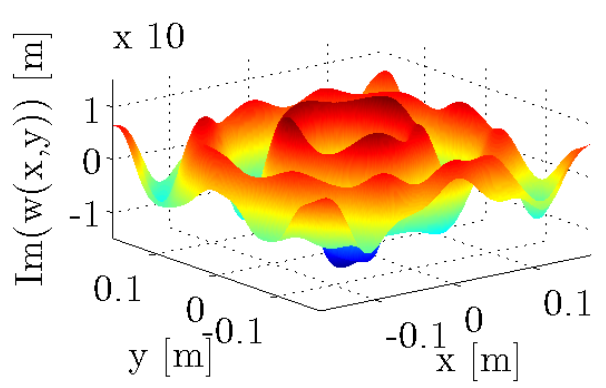

Fig. 13. Filtered complex "infinite" plate displacement at 1503.2 Hz: (a)-(b) ideal 2D filtered, (c)(d) 2D Butterworth filtered. 
(a)

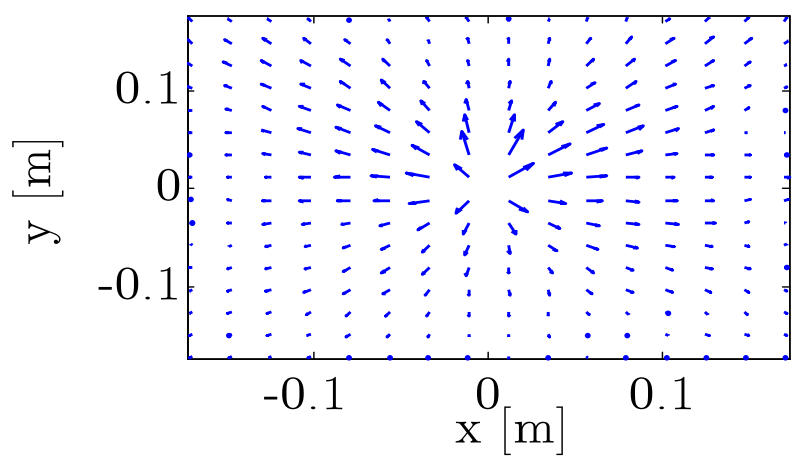

(b)

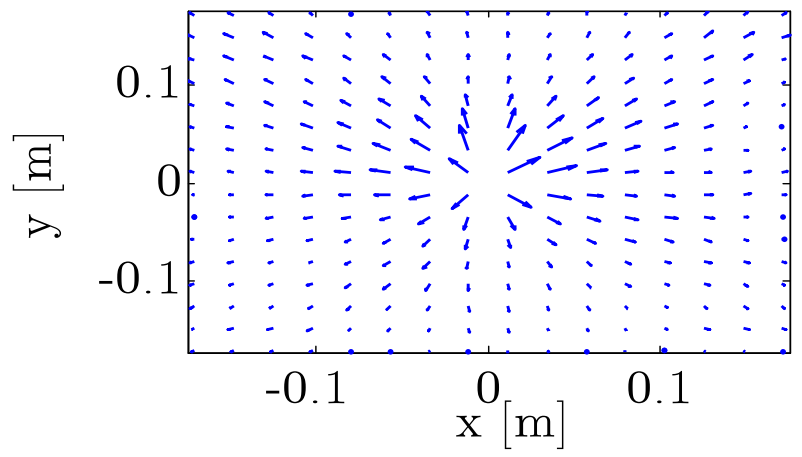

Fig. 14. Filtered active vibrational energy flow maps of the "infinite" plate displacement at 1503.2 Hz: (a) ideal filter; (b) Butterworth filter. Magnitude of the maximum energy flow vector shown represents approximately $10^{-4} \mathrm{~W} / \mathrm{m}$. 

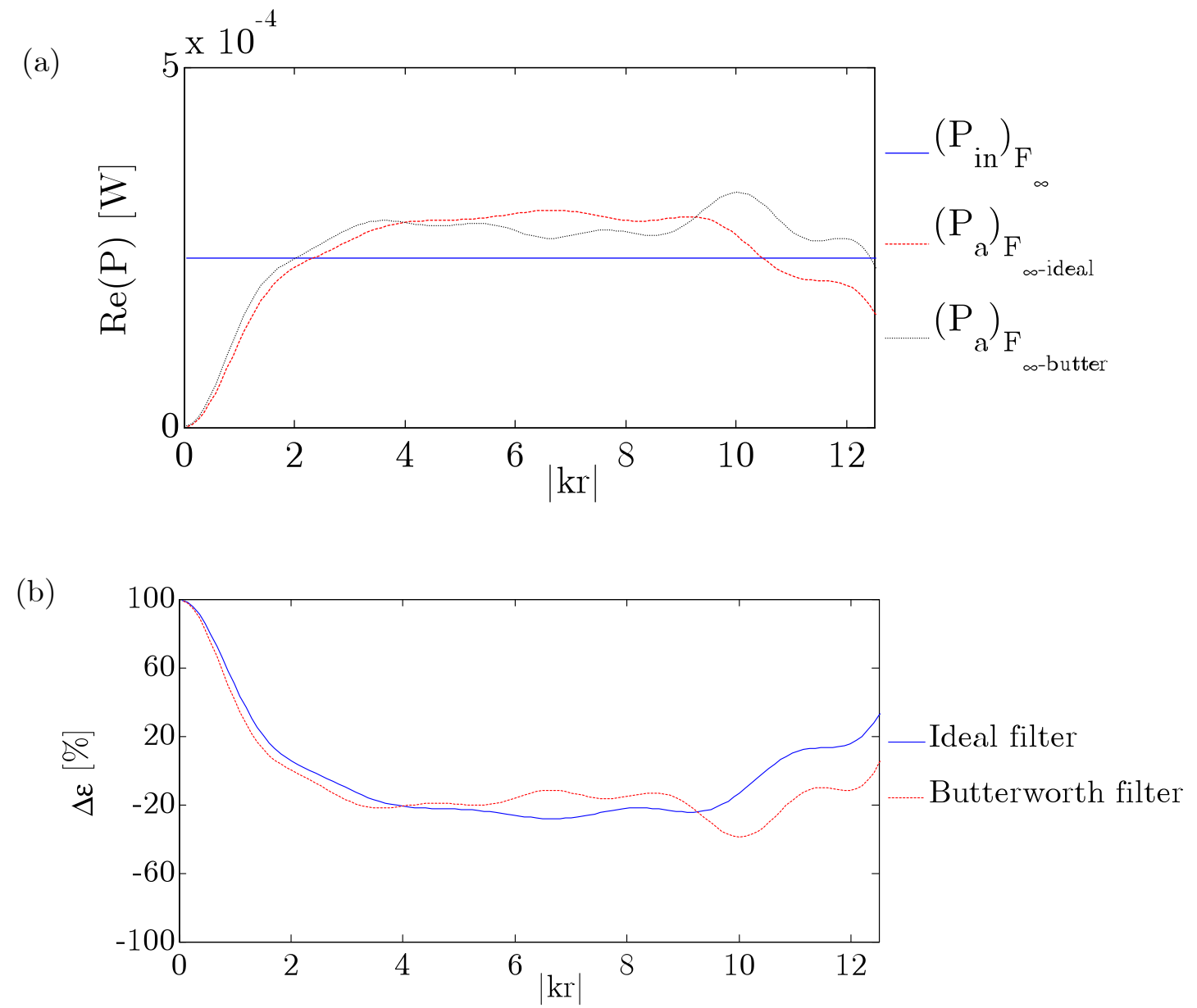

Fig. 15. Comparison of the total transmitted ESPI measured vibrational power and the vibrational input power at $1503.2 \mathrm{~Hz}$ : (a) power in Watts; (b) relative difference in percent. 
(a)

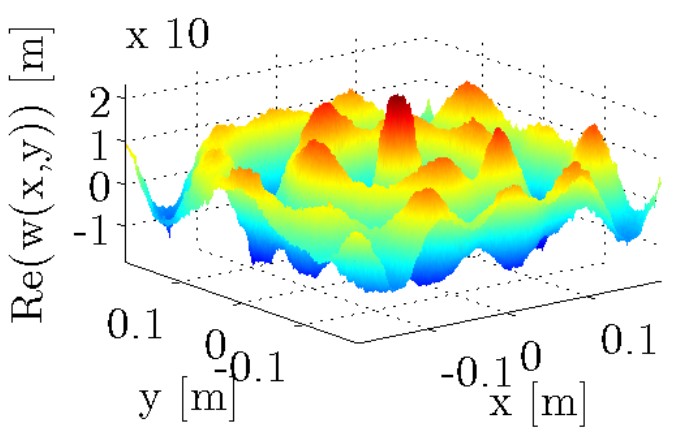

(b)

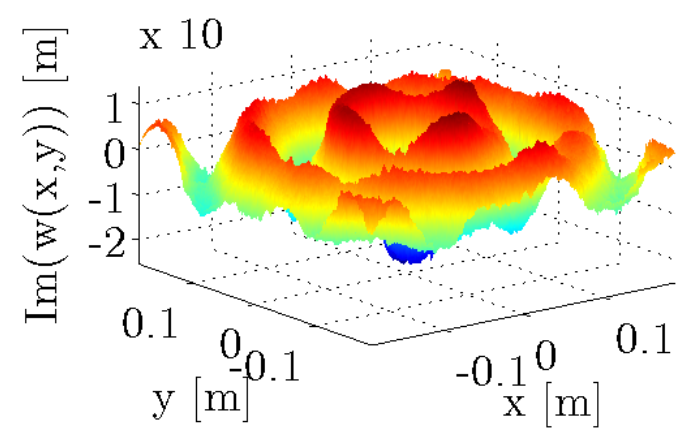

(c)

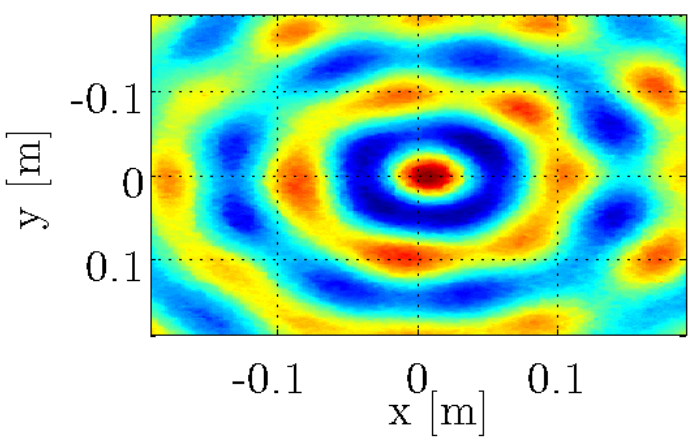

(d)

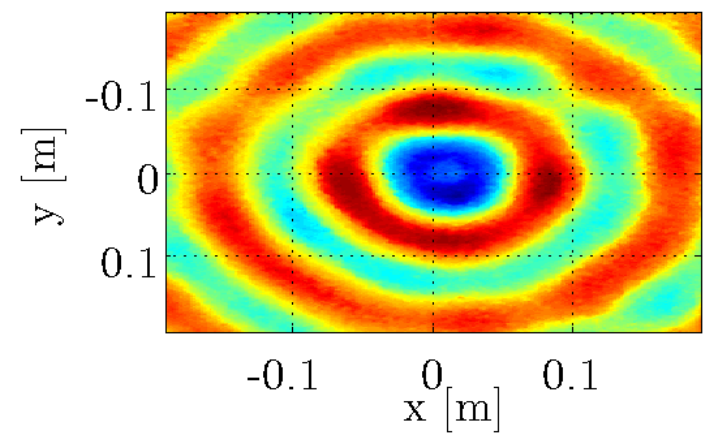

Fig. 16. ESPI image of the measured and truncated "infinite" plate displacement at $1194.4 \mathrm{~Hz}$ (a) 2D real part; (b) 2D imaginary part; (c) top view real part; (d) top view imaginary part. 
(a)

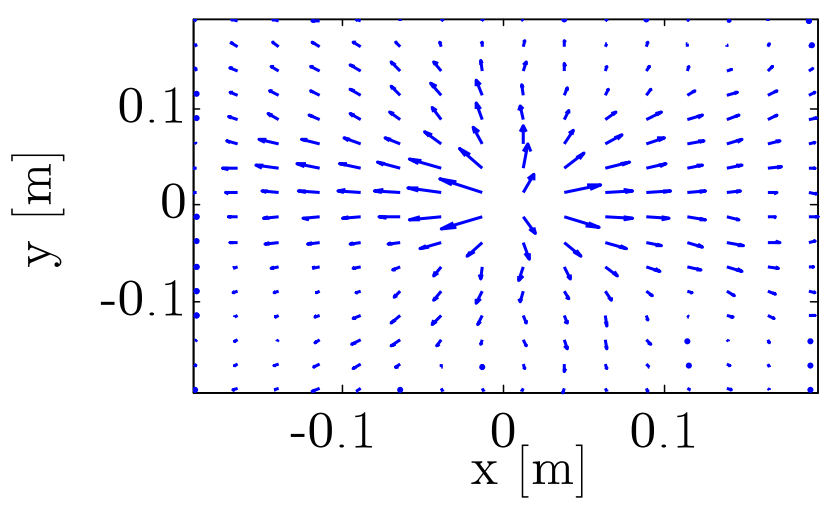

(b)

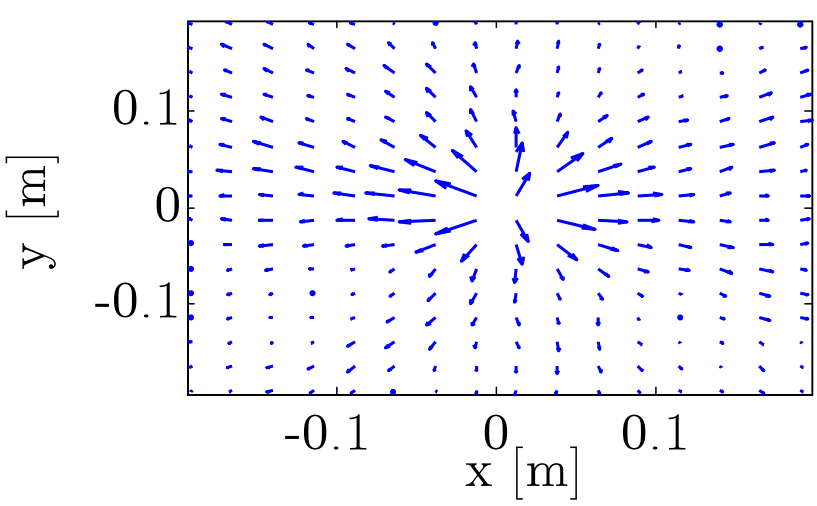

Fig. 17. Filtered active vibrational energy flow maps of the "infinite" plate displacement at 1194.4 Hz: (a) ideal filter; (b) Butterworth filter. Magnitude of the maximum energy flow vector shown represents approximately $5 \cdot 10^{-5} \mathrm{~W} / \mathrm{m}$. 
(a)

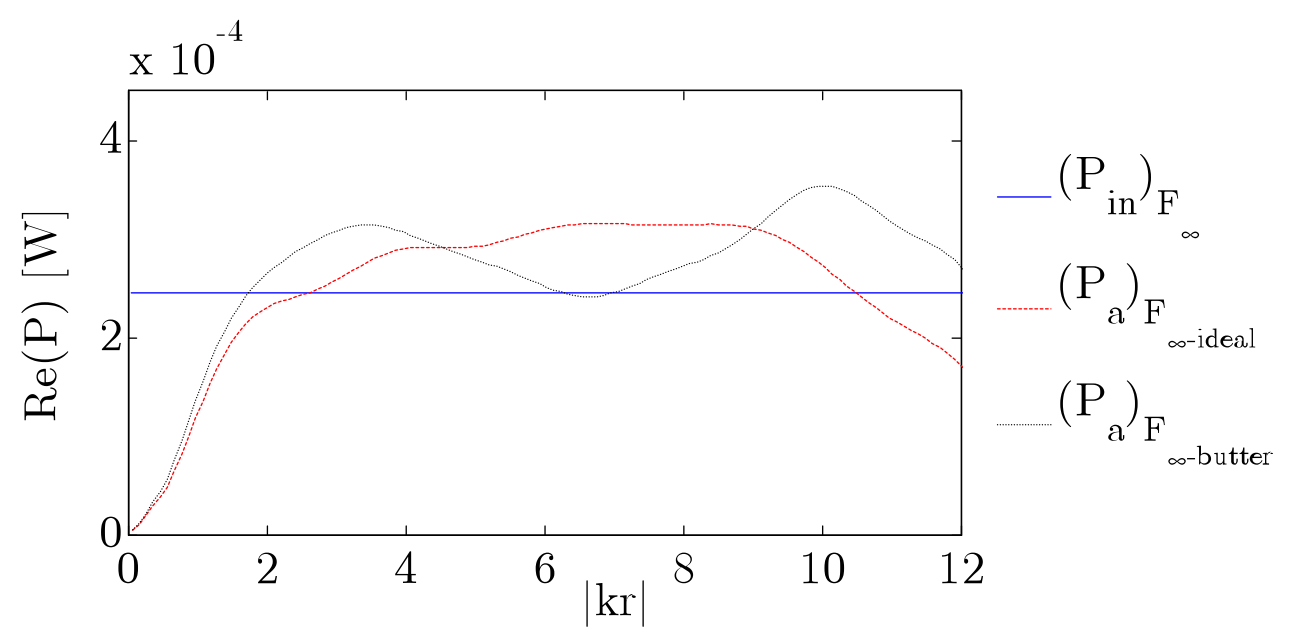

(b)

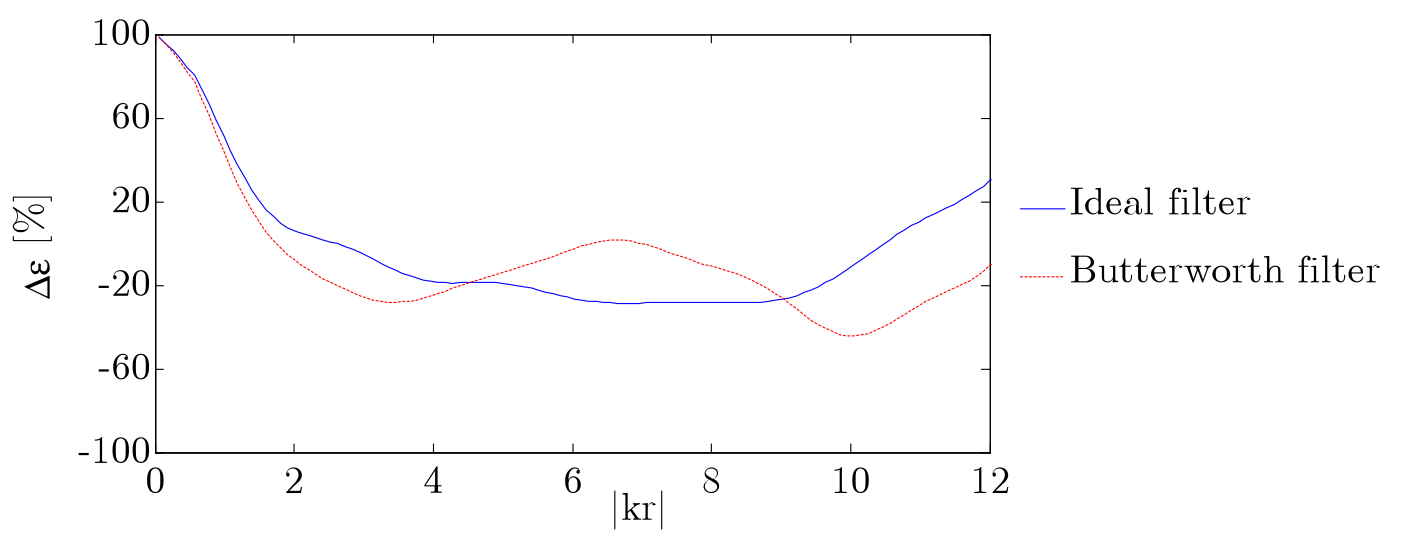

Fig. 18. Comparison of the total transmitted ESPI measured vibrational power and the vibrational input power at $1194.4 \mathrm{~Hz}$ : (a) power in Watts; (b) relative difference in percent. 
(a)

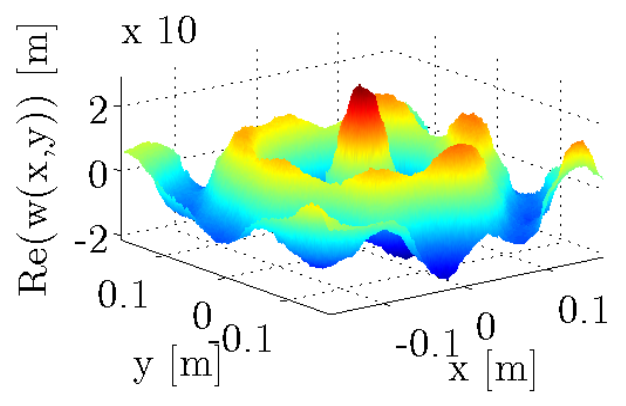

(b)

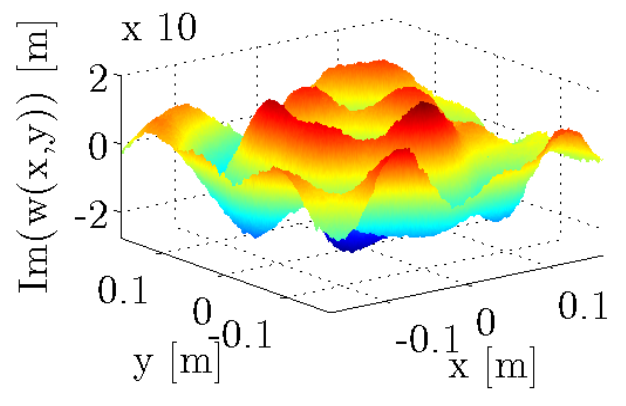

(c)

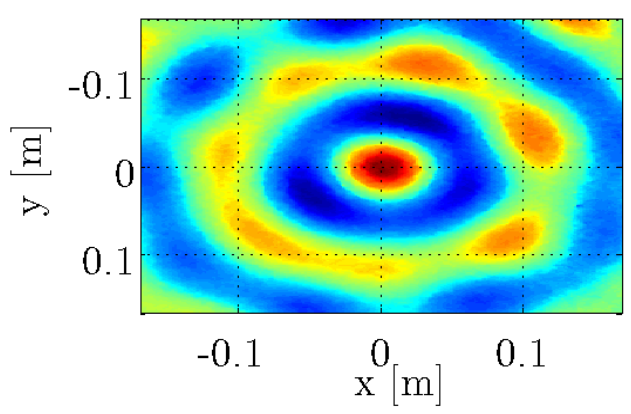

(d)

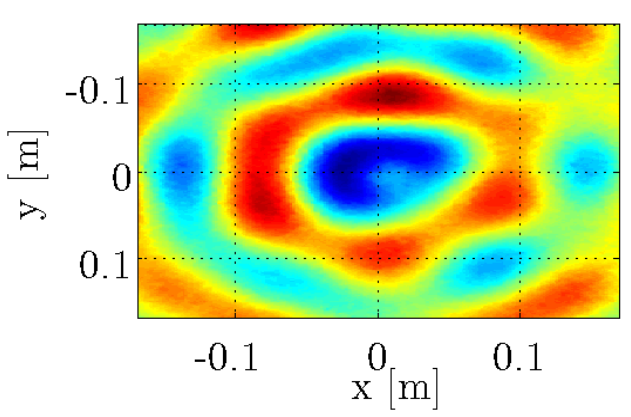

Fig. 19. ESPI image of the measured and truncated "infinite" plate displacement at $899.5 \mathrm{~Hz}$ : (a) 2D real part; (b) 2D imaginary part; (c) top view real part; (d) top view imaginary part. 
(a)

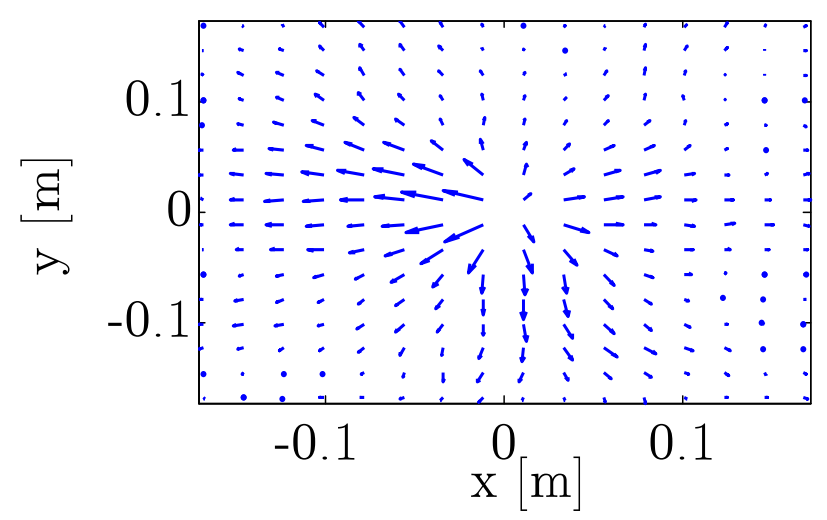

(b)

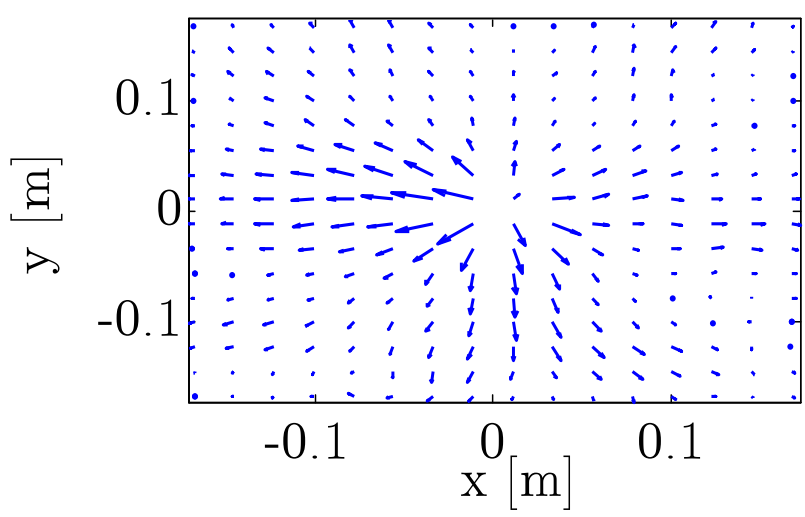

Fig. 20. Filtered active vibrational energy flow maps of the "infinite" plate displacement at 899.5 Hz: (a) ideal filter; (b) Butterworth filter. Magnitude of the maximum energy flow vector shown represents approximately $8 \cdot 10^{-5} \mathrm{~W} / \mathrm{m}$. 

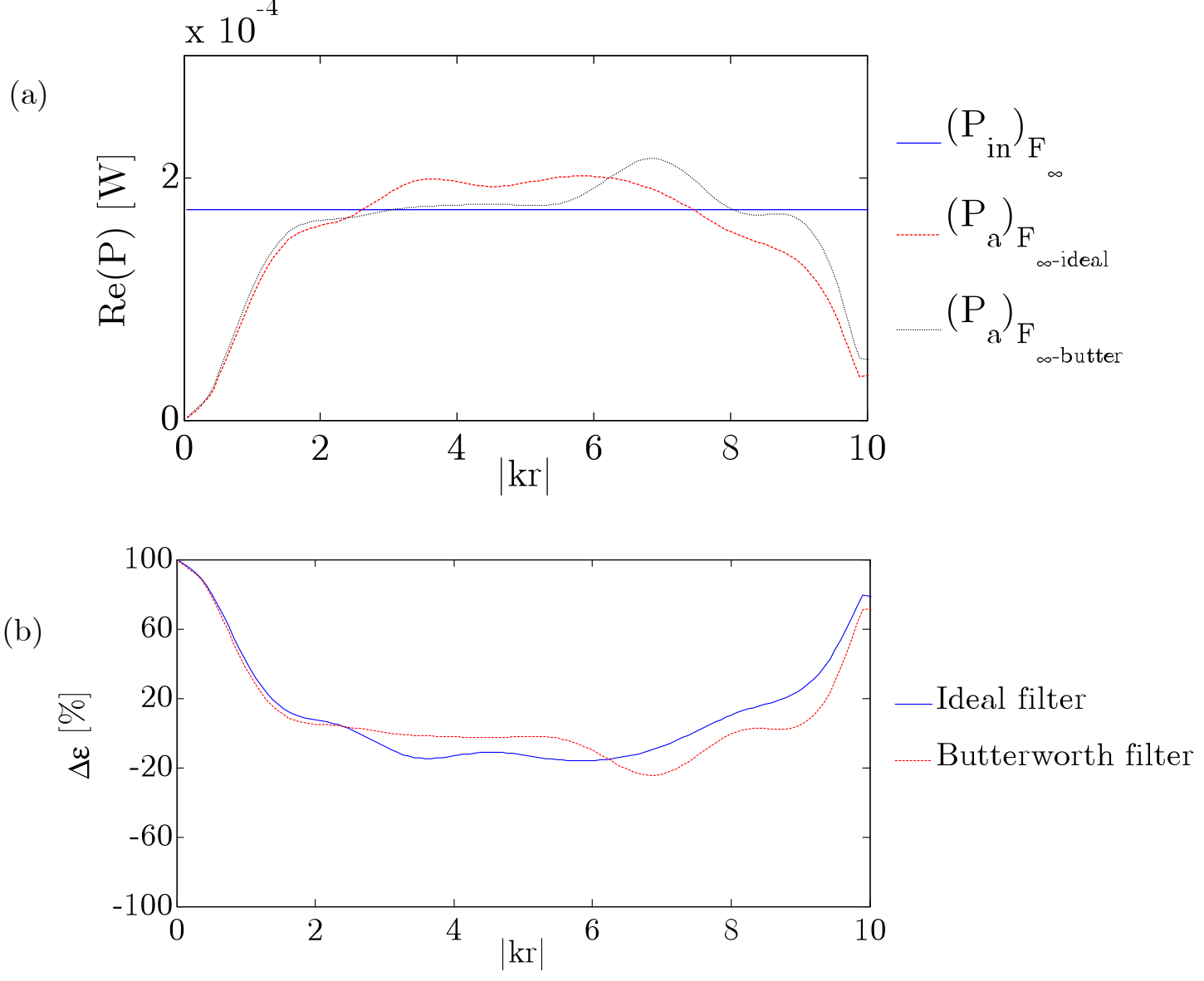

Fig. 21. Comparison of the total transmitted ESPI measured vibrational power and the vibrational input power at $899.5 \mathrm{~Hz}$ : (a) power in Watts; (b) relative difference in percent. 
(a)

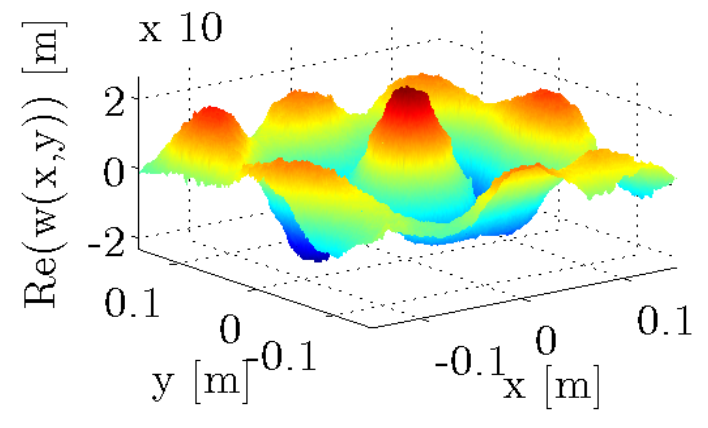

(b)

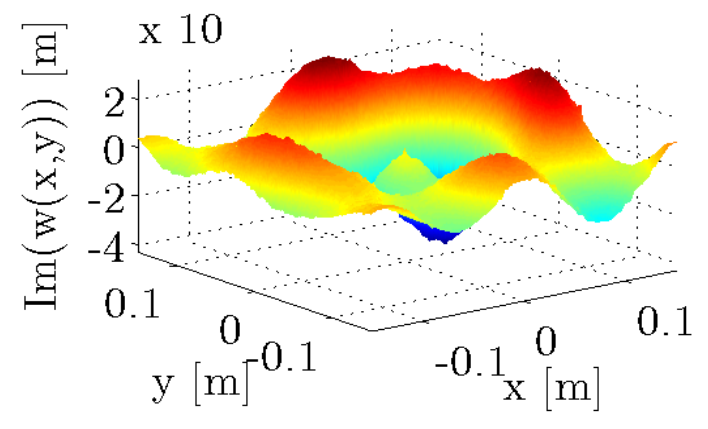

(c)

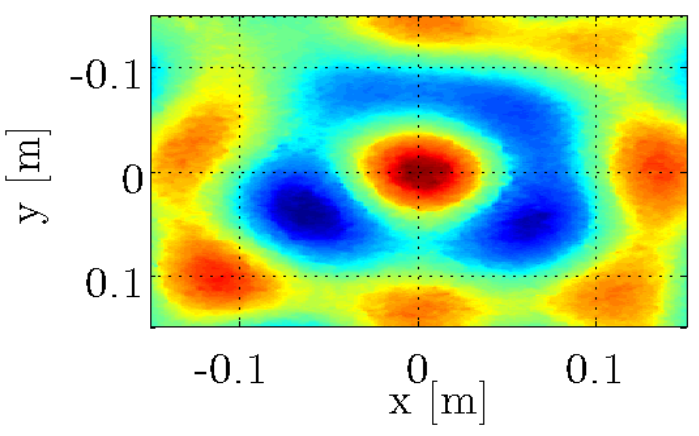

(d)

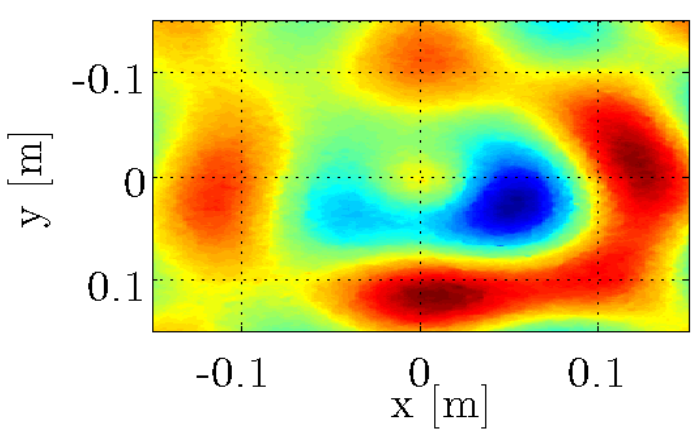

Fig. 22. ESPI image of the measured and truncated "infinite" plate displacement at $605.6 \mathrm{~Hz}$ : (a) 2D real part; (b) 2D imaginary part; (c) top view real part; (d) top view imaginary part. 
(a)

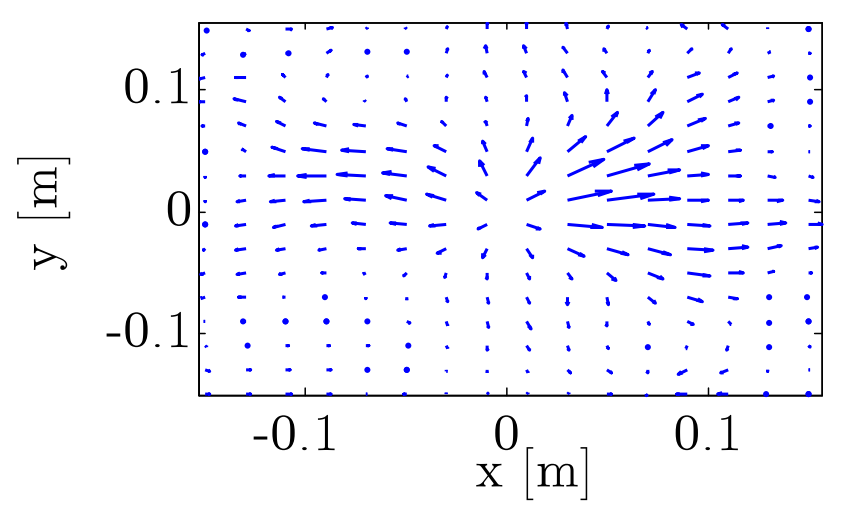

(b)

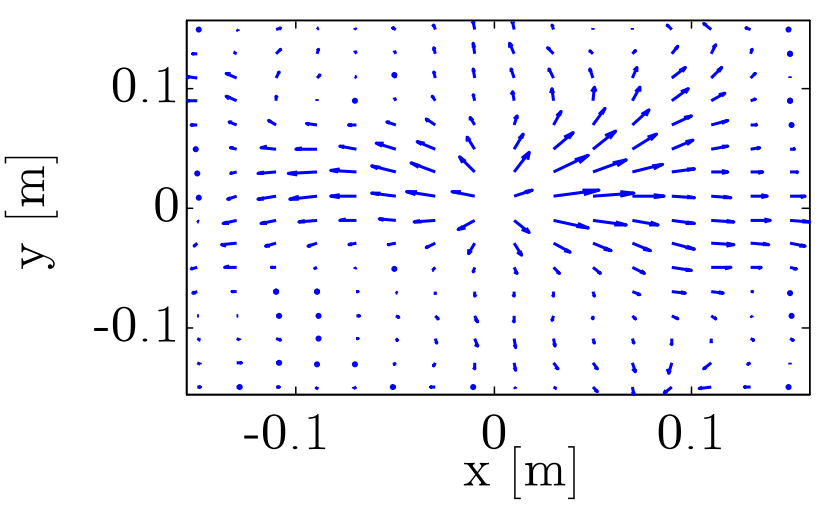

Fig. 23. Filtered active vibrational energy flow maps of the "infinite" plate displacement at 605.6 Hz: (a) ideal filter; (b) Butterworth filter. Magnitude of the maximum energy flow vector shown represents approximately $3 \cdot 10^{-5} \mathrm{~W} / \mathrm{m}$. 
(a)

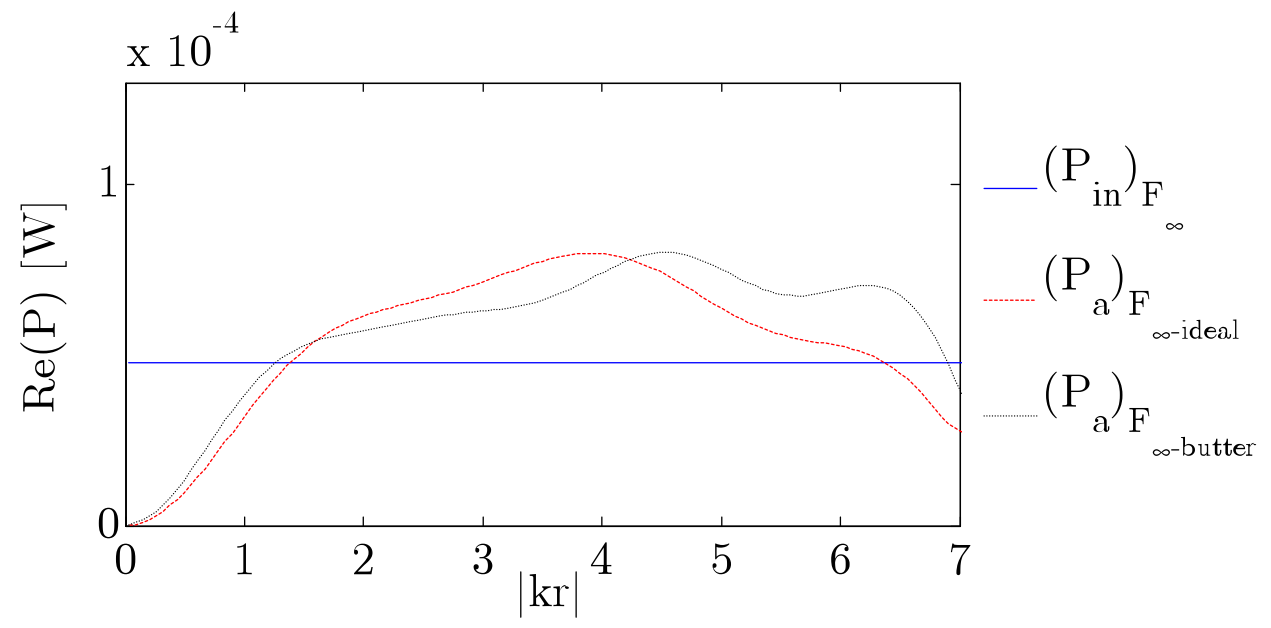

(b)

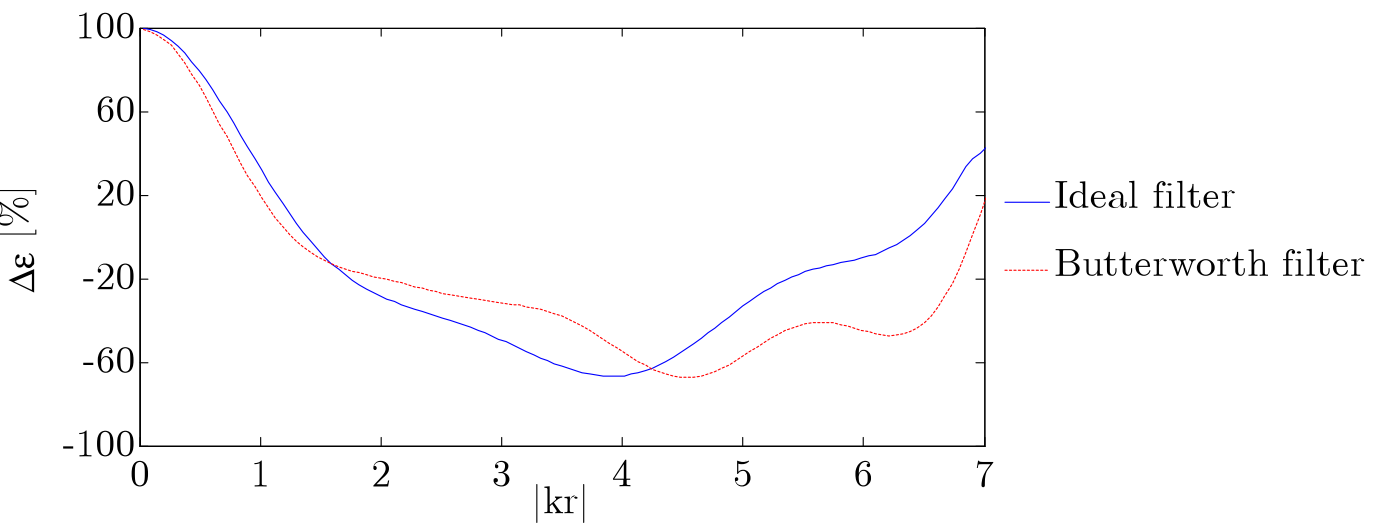

Fig. 24. Comparison of the total transmitted ESPI measured vibrational power and the vibrational input power at $605.6 \mathrm{~Hz}$ : (a) power in Watts; (b) relative difference in percent. 


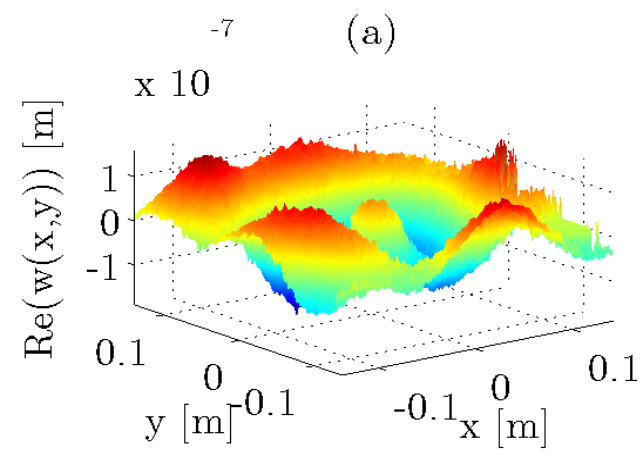

$-7$

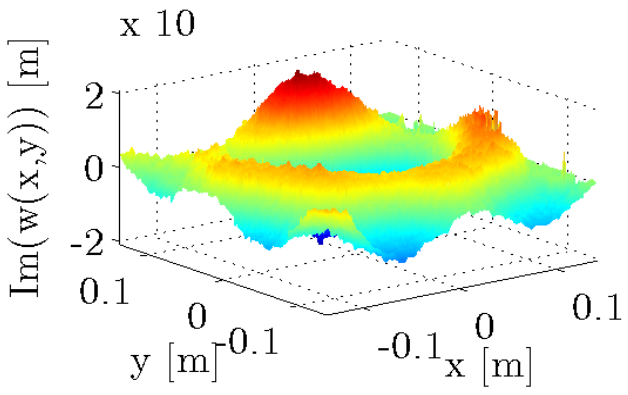

(c)

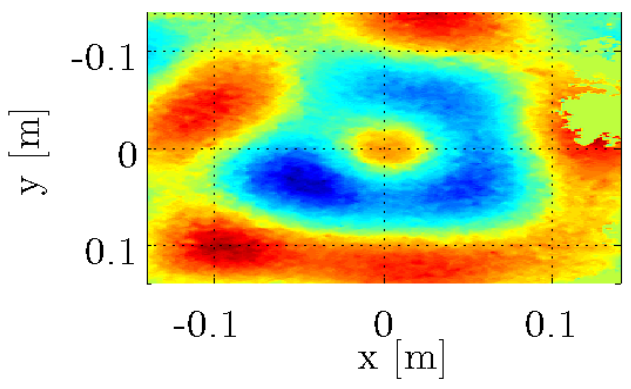

(d)

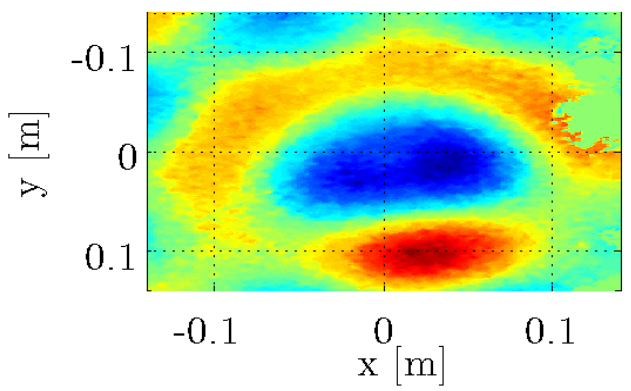

Fig. 25. ESPI image of the measured and truncated "infinite" plate displacement at $569.7 \mathrm{~Hz}$ : (a) $2 \mathrm{D}$ real part; (b) 2D imaginary part; (c) top view real part; (d) top view imaginary part. 
(a)

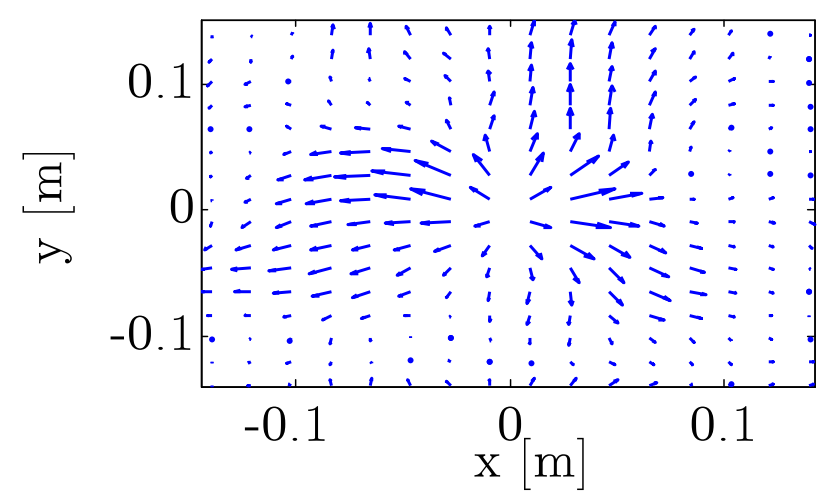

(b)

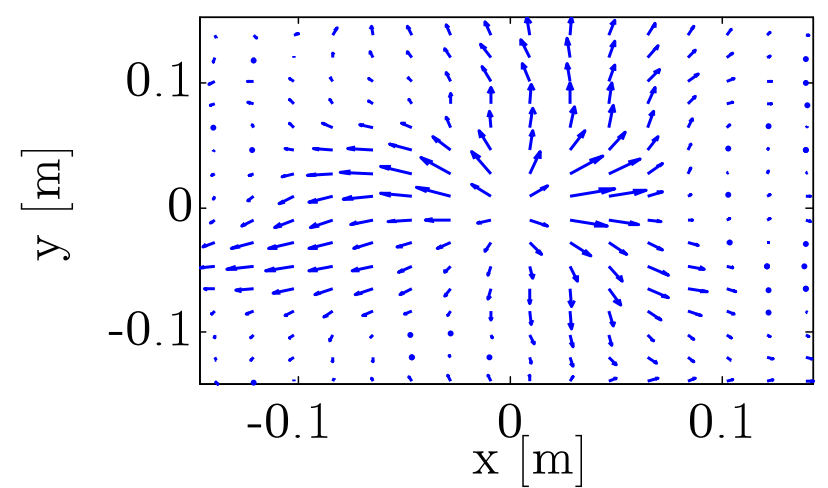

Fig. 26. Filtered active vibrational energy flow maps of the "infinite" plate displacement at 569.7 Hz: (a) ideal filter; (b) Butterworth filter. Magnitude of the maximum energy flow vector shown represents approximately $8 \cdot 10^{-6} \mathrm{~W} / \mathrm{m}$. 


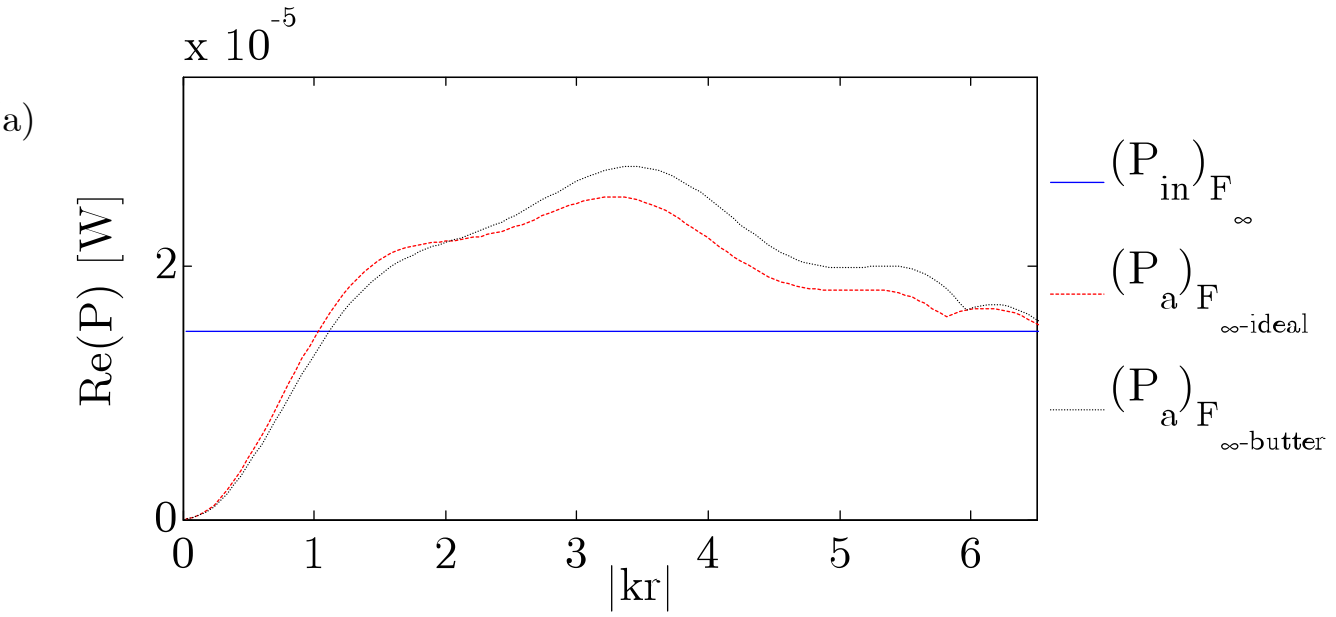

(b)

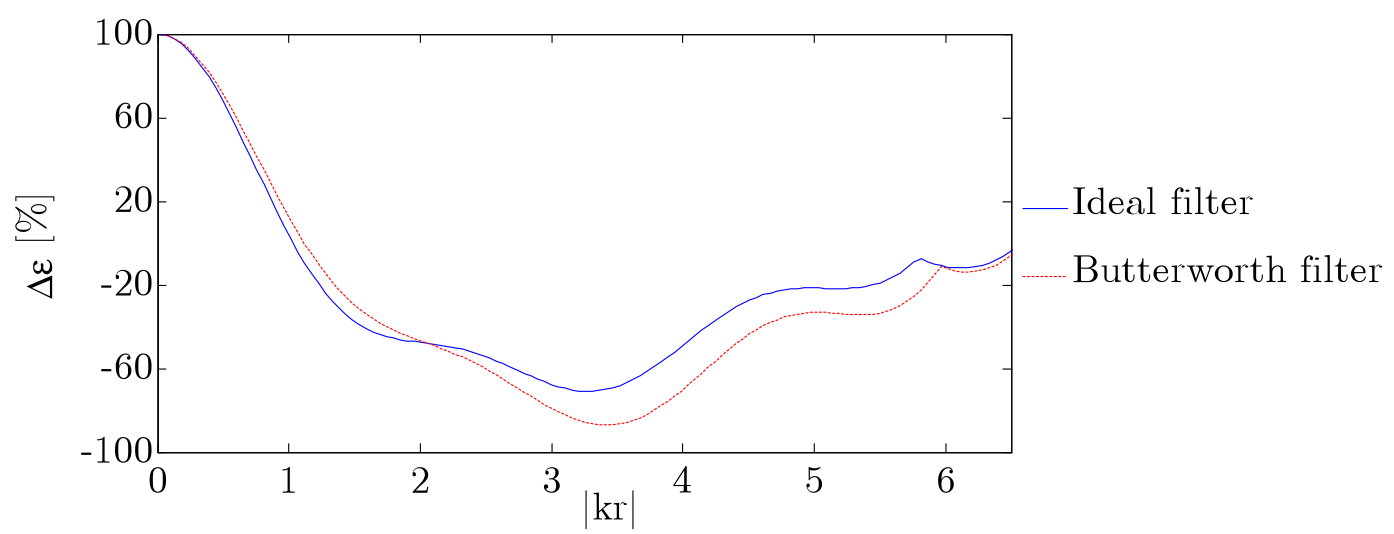

Fig. 27. Comparison of the total transmitted ESPI measured vibrational power and the vibrational input power at $569.7 \mathrm{~Hz}$ : (a) power in Watts; (b) relative difference in percent. 
(a)
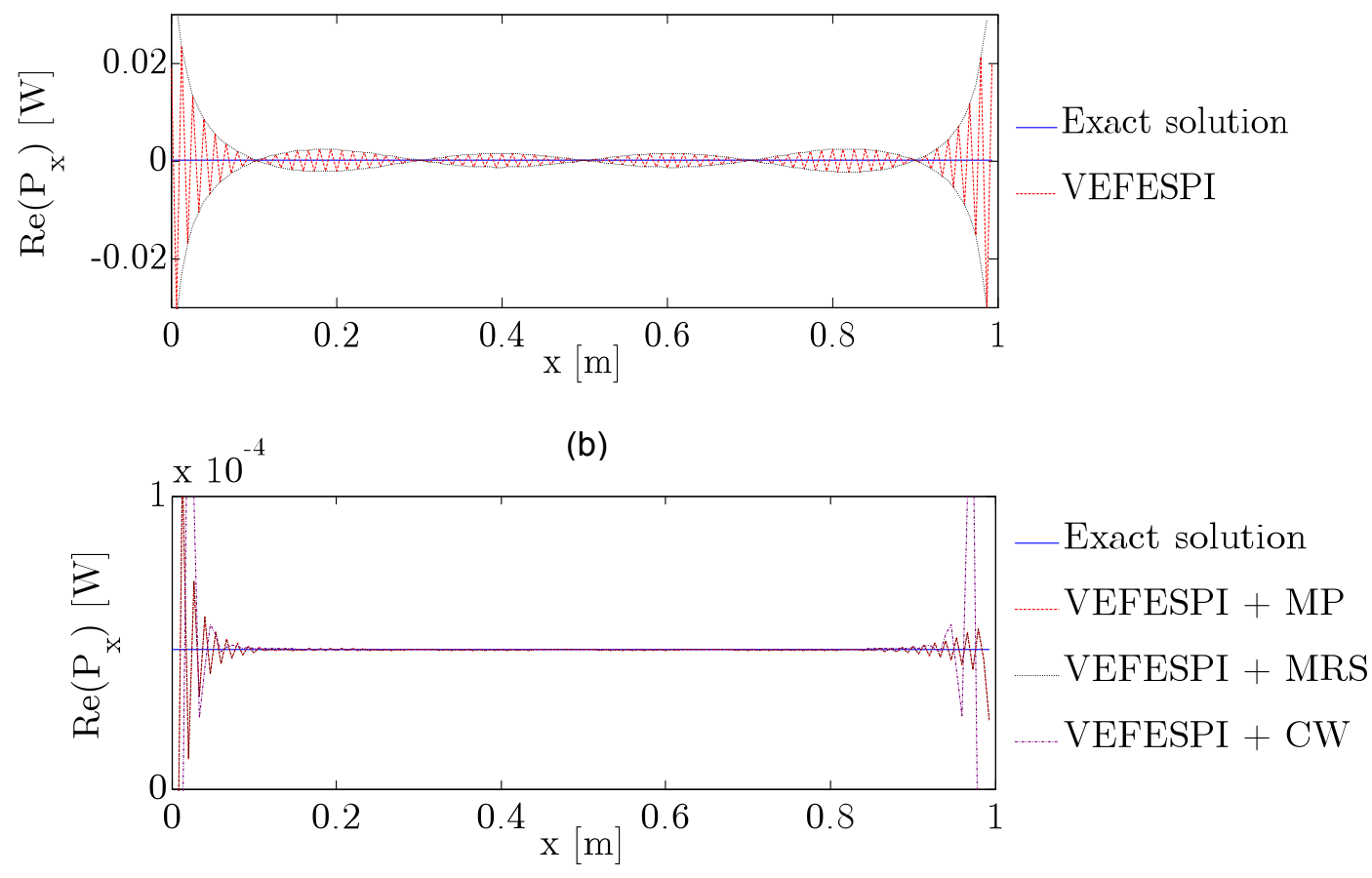

Fig. A1. Comparison of the input power (Exact) and VEFESPI determined transmitted power of an infinite beam displacement: (a) VEFESPI with non-periodic beam displacement data; (b) VEFESPI with MP, MRS and CW techniques, respectively. 


\section{Table 1}

"Infinite" plate material and geometric properties.

\begin{tabular}{ll}
\hline Young's modulus & $E=2.07 \cdot 10^{11} \mathrm{~N} / \mathrm{m}^{2}$ \\
\hline mass density & $\rho=7.85 \cdot 10^{3} \mathrm{~kg} / \mathrm{m}^{3}$ \\
\hline plate thickness & $h=1 \cdot 10^{-3} \mathrm{~m}$ \\
\hline Poisson's ratio & $v=0.33$ \\
\hline
\end{tabular}




\section{Table 2}

Measured vibrational input power values (in Watts).

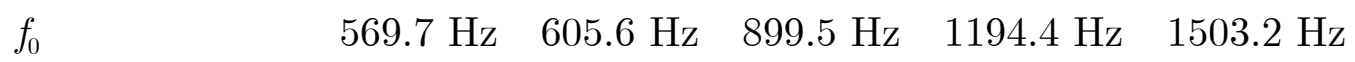

\begin{tabular}{llllll}
\hline $\begin{array}{l}\text { Theoretical impedance based } \\
\text { power measurement, }\left(P_{\text {in }}\right)_{F_{\sharp}}\end{array}$ & $1.49 \cdot 10^{-5}$ & $4.79 \cdot 10^{-5}$ & $1.73 \cdot 10^{-4}$ & $2.45 \cdot 10^{-4}$ & $2.35 \cdot 10^{-4}$ \\
\hline
\end{tabular}

\begin{tabular}{|c|c|c|}
\hline Experimental mobility & $1.14 \cdot 10^{-5}$ & $3.78 \cdot 10^{-5}$ \\
\hline
\end{tabular}

